\title{
Encyclopedia of Database Systems
}


Ling Liu $•$ M. Tamer Özsu

Editors

\section{Encyclopedia of Database Systems}

Second Edition

With 1374 Figures and 143 Tables

算 Springer 


\section{Editors}

\section{Ling Liu}

Georgia Institute of Technology College

of Computing

Atlanta, GA, USA

\author{
M. Tamer Özsu \\ University of Waterloo School of Computer Science \\ Waterloo, ON, Canada
}

ISBN 978-1-4614-8266-6 ISBN 978-1-4614-8265-9 (eBook)

ISBN 978-1-4614-8264-2 (print and electronic bundle)

https://doi.org/10.1007/978-1-4614-8265-9

Library of Congress Control Number: 2018938558

1st edition: (C) Springer Science+Business Media, LLC 2009 (USA)

(C) Springer Science+Business Media, LLC, part of Springer Nature 2018

This work is subject to copyright. All rights are reserved by the Publisher, whether the whole or part of the material is concerned, specifically the rights of translation, reprinting, reuse of illustrations, recitation, broadcasting, reproduction on microfilms or in any other physical way, and transmission or information storage and retrieval, electronic adaptation, computer software, or by similar or dissimilar methodology now known or hereafter developed.

The use of general descriptive names, registered names, trademarks, service marks, etc. in this publication does not imply, even in the absence of a specific statement, that such names are exempt from the relevant protective laws and regulations and therefore free for general use.

The publisher, the authors and the editors are safe to assume that the advice and information in this book are believed to be true and accurate at the date of publication. Neither the publisher nor the authors or the editors give a warranty, express or implied, with respect to the material contained herein or for any errors or omissions that may have been made. The publisher remains neutral with regard to jurisdictional claims in published maps and institutional affiliations.

Printed on acid-free paper

This Springer imprint is published by the registered company Springer Science+Business Media, LLC part of Springer Nature.

The registered company address is: 233 Spring Street, New York, NY 10013, U.S.A. 


\section{Preface to the Second Edition}

Since the release of the first volume of this Encyclopedia, big data has emerged as a central feature of information technology innovation in many business, science, and engineering fields. Databases are one of the fundamental core technologies for big data systems and big data analytics. In order to extract features and derive values from big data, it must be stored, processed, and analyzed in a timely manner. Not surprisingly, big data not only fuels the development and deployment of database systems and database technologies, it also opens doors to new opportunities and new challenges in the field of databases. As data grows in volume, velocity, variety, and with the attendant veracity issues, there is a growing demand for volume-scalable databases, velocity-adaptive databases, and variety-capable databases that can handle data quality issues properly. As machine learning and artificial intelligence renew their momentum with the power of big data, there is an increasing demand for new generation of database systems that are built for extracting features from databases as efficient and effective as conventional database systems are capable of for querying databases.

The first edition of the Encyclopedia of Database Systems is a comprehensive, multivolume collection of over 1,250 in-depth entries $(3,067$ including synonyms), covering important concepts on all aspects of database systems, including areas of current interest and research results of historical significance. This second edition of Encyclopedia of Database Systems expands the first edition by enriching the content of existing entries, expanding existing topic areas with new entries, adding a set of cutting-edge topic areas, including cloud data management, crowdsourcing, data analytics, data provenance management, graph data management, social networks, and uncertain data management to name a few. The new entries and the new topic areas were determined through discussions and consultations with the Advisory Board of the Encyclopedia of Database Systems. Each of the new topic areas was managed by a new Area Editor who, together with the editorin-chief, further developed the content for each area, soliciting experts in the field as contributors to write the entries, and performed the necessary technical editing. We also reviewed the entries from the first edition and revised them as needed to bring them up-to-date. 
We would like to thank the members of the Advisory Board, the Editorial Board, and all of the authors for their contributions to this second edition. We would also like to thank Springer's editors and staff, including Susan Lagerstrom-Fife, Michael Hermann, and Sonja Peterson for their assistance and support throughout the project, and Annalea Manalili for her involvement in the early period of this project.

In closing, we trust the Encyclopedia can serve as a valuable source for students, researchers, and practitioners who need a quick and authoritative reference to the subject on database systems. Suggestions and feedbacks to further improve the Encyclopedia are welcome from readers and from the community. 


\section{Preface to the First Edition}

We are in an information era where generating and storing large amounts of data are commonplace. A growing number of organizations routinely handle terabytes and exabytes of data, and individual digital data collections easily reach multiple gigabytes. Along with the increases in volume, the modality of digitized data that requires efficient management and the access modes to these data have become more varied. It is increasingly common for business and personal data collections to include images, video, voice, and unstructured text; the retrieval of these data comprises various forms, including structured queries, keyword search, and visual access. Data have become a highly valued asset for governments, industries and individuals, and the management of these data collections remains a critical technical challenge.

Database technology has matured over the past four decades and is now quite ubiquitous in many applications that deal with more traditional business data. The challenges of expanding data management to include other data modalities while maintaining the fundamental tenets of database management (data independence, data integrity, data consistency, etc.) are issues that the community continues to work on. The lines between database management and other fields such as information retrieval, multimedia retrieval, and data visualization are increasingly blurred.

This multi-volume Encyclopedia of Database Systems provides easy access to important concepts on all aspects of database systems, including areas of current interest and research results of historical significance. It is a comprehensive collection of over 1,250 in-depth entries (3,067 including synonyms) that present coverage of the important concepts, issues, emerging technology and future trends in the field of database technologies, systems, and applications. The content of the Encyclopedia was determined through wide consultations. We were assisted by an Advisory Board in coming up with the overall structure and content. Each of these areas were put under the control of Area Editors (70 in total) who further developed the content for each area, soliciting experts in the field as contributors to write the entries, and performed the necessary technical editing. Some of them even wrote entries themselves. Nearly 1,000 authors were involved in writing entries. 
The intended audience for the Encyclopedia is technically broad and diverse. It includes anyone concerned with database system technology and its applications. Specifically, the Encyclopedia can serve as a valuable and authoritative reference for students, researchers and practitioners who need a quick and authoritative reference to the subject of databases, data management, and database systems. We anticipate that many people will benefit from this reference work, including database specialists, software developers, scientists and engineers who need to deal with (structured, semi-structured or unstructured) large datasets. In addition, database and data mining researchers and scholars in the many areas that apply database technologies, such as artificial intelligence, software engineering, robotics and computer vision, machine learning, finance and marketing are expected to benefit from the Encyclopedia.

We would like to thank the members of the Advisory Board, the Editorial Board, and the individual contributors for their help in creating this Encyclopedia. The success of the Encyclopedia could not have been achieved without the expertise and the effort of the many contributors. Our sincere thanks also go to Springer's editors and staff, including Jennifer Carlson, Susan Lagerstrom-Fife, Oona Schmid, and Susan Bednarczyk for their support throughout the project.

Finally, we would very much like to hear from readers for any suggestions regarding the Encyclopedia's content. With a project of this size and scope, it is quite possible that we may have missed some concepts. It is also possible that some entries may benefit from revisions and clarifications. We are committed to issuing periodic updates and we look forward to the feedback from the community to improve the Encyclopedia.

Ling Liu

M. Tamer Özsu 


\section{List of Topics}

Peer-to-Peer Data Management

\section{Section Editor: Karl Aberer}

Approximate Queries in Peer-to-Peer Systems

Declarative Networking

Distributed Hash Table

Load Balancing in Peer-to-Peer Overlay

Networks

Overlay Network

Peer Data Management System

Peer-to-Peer Content Distribution

Peer-to-Peer Data Integration

Peer-to-Peer Overlay Networks: Structure,

Routing and Maintenance

Peer-to-Peer Publish-Subscribe Systems

Peer-to-Peer Storage

Peer-to-Peer System

Reputation and Trust

Semantic Overlay Networks

Social Networks

Structured Data in Peer-to-Peer Systems

Trust and Reputation in Peer-to-Peer Systems

Updates and Transactions in Peer-to-Peer

Systems

\section{Database Management System}

Architectures

\section{Section Editor: Anastasia Ailamaki}

Application Server

Architecture-Conscious Database System

Buffer Manager

Buffer Pool

Cache-Conscious Query Processing

Client-Server Architecture

Concurrency Control Manager
Connection

Data Partitioning

Database Management System

DBMS Component

DBMS Interface

Disk

Distributed DBMS

Execution Skew

Inter-query Parallelism

Intra-query Parallelism

Locality

Logging/Recovery Subsystem

Main Memory DBMS

Main Memory

Memory Hierarchy

Memory Locality

Multitier Architecture

Operator-Level Parallelism

Pipeline

Process Structure of a DBMS

Processor Cache

Query Processor

Session

Staged DBMS

Stop-\&-Go Operator

Storage Manager

Transaction Manager

Information Retrieval Models

Section Editor: Giambattista Amati

Anchor Text

BM25

Boolean Model

Divergence-from-Randomness Models

Document Field 
Document Length Normalization

Document Links and Hyperlinks

Entity Resolution

Field-Based Information Retrieval Models

Fuzzy Models

Information Retrieval Models

Information Retrieval

Inverse Document Frequency

Language Models

Logical Models of Information Retrieval

N-Gram Models

Probabilistic Retrieval Models and Binary

Independence Retrieval (BIR) Model

Probability Ranking Principle

Probability Smoothing

Query Expansion Models

Rocchio's Formula

Term Proximity

Time and Information Retrieval

Two-Poisson Model

Vector-Space Model

WEB Information Retrieval Models

\section{XML Data Management}

\section{Section Editor: Sihem Amer-Yahia}

Active XML

Application Benchmark

Microbenchmark

Ranked XML Processing

Semi-structured Data

XML Access Control

XML Attribute

XML Benchmarks

XML Compression

XML Document

XML Element

XML Indexing

XML Information Integration

XML Integrity Constraints

XML Parsing, SAX/DOM

XML Programming

XML Publish/Subscribe

XML Publishing

XML Schema

XML Selectivity Estimation

XML Storage

XML Tree Pattern, XML Twig Query
XML Tuple Algebra

XML Typechecking

XML Types

XML Updates

XML Views

$\mathrm{XML}$

XPath/XQuery

XQuery Full-Text

XQuery Processors

XSL/XSLT

Database Middleware

Section Editor: Cristiana Amza

Adaptive Middleware for Message Queuing Systems

Database Middleware

Java Enterprise Edition

Mediation

Message Queuing Systems

Middleware Support for Database Replication and Caching

Middleware Support for Precise Failure

Semantics

Replication in Multitier Architectures

Transactional Middleware

Database Management Utilities

Section Editor: Philippe Bonnet

Administration Wizards

Performance Monitoring Tools

Visual Interfaces

Section Editor: Tiziana Catarci

Adaptive Interfaces

Browsing

Diagram

Direct Manipulation

Dynamic Web Pages

eAccessibility

Form

Human-Computer Interaction

Icon

Information Foraging

Information Navigation

Mobile Interfaces 
Multimedia Presentation Databases

Multimodal Interfaces

Natural Interaction

Ontology Visual Querying

Result Display

Social Applications

Temporal Visual Languages

Usability

Visual Formalisms

Visual Interaction

Visual Interfaces for Geographic Data

Visual Interfaces

Visual Metaphor

Visual Perception

Visual Query Languages for Data Stream

Visual Query Language

Visual Representation

Web Mashups

WIMP Interfaces

\section{Stream Data Management}

\section{Section Editor: Ugur Cetintemel}

Adaptive Stream Processing

Big Stream Systems

Continuous Query

Data Stream Management Architectures and Prototypes

Data Stream

Distributed Data Streams

Event and Pattern Detection over Streams

Fault Tolerance and High Availability in

Data Stream Management Systems

Load Shedding

Nonrelational Streams

One-Pass Algorithm

Publish/Subscribe Over Streams

Punctuations

Scheduling Strategies for Data Stream

Processing

Stream Processing on Modern Hardware

Stream Processing

Stream-Oriented Query Languages

and Operators

Streaming Applications

Text Stream Processing

Transactional Stream Processing

Window-Based Query Processing
Windows

XML Stream Processing

Querying over Data Integration Systems

Section Editor: Kevin Chang

Information Integration

Ontology-Based Data Access and Integration

Query Processing in Data Integration Systems

Query Translation

Rewriting Queries Using Views

View-Based Data Integration

Self-Management

\section{Section Editor: Surajit Chaudhuri}

Database Tuning Using Combinatorial Search

Database Tuning Using Online Algorithms

Database Tuning Using Trade-Off Elimination

Self-Management Technology in Databases

\section{Text Mining}

\section{Section Editor: Zheng Chen}

Cross-Language Mining and Retrieval

Information Extraction

Opinion Mining

Text Categorization

Text Clustering

Text Generation

Text Indexing and Retrieval

Text Mining

Text Representation

Text Segmentation

Text Semantic Representation

Text Streaming Model

Text Summarization

Text Visualization

Topic Detection and Tracking

Trust in Blogosphere

\section{Extended Transaction Models}

Section Editor: Panos K. Chrysanthis

Compensating Transactions

ConTract

Correctness Criteria Beyond Serializability

E-Commerce Transactions 
Extended Transaction Models and the ACTA

Framework

Flex Transactions

Generalization of ACID Properties

Multilevel Transactions and Object-Model

Transactions

Nested Transaction Models

Open Nested Transaction Models

Polytransactions

Real-Time Transaction Processing

Sagas

Semantic Atomicity

Semantics-Based Concurrency Control

Split Transactions

Transactional Processes

Web Transactions

Workflow Transactions

\section{Privacy-Preserving Data Mining}

\section{Section Editor: Chris Clifton}

Horizontally Partitioned Data

Individually Identifiable Data

Matrix Masking

Privacy Metrics

Privacy-Preserving Data Mining

Randomization Methods to Ensure

Data Privacy

Secure Multiparty Computation Methods

Statistical Disclosure Limitation for

Data Access

Vertically Partitioned Data

\section{Digital Libraries}

\section{Section Editor: Amr El Abbadi}

Browsing in Digital Libraries

Cataloging in Digital Libraries

Citation

Copyright Issues in Databases

Dewey Decimal System

Digital Archives and Preservation

Digital Curation

Digital Libraries

European Law in Databases

Gazetteers

Georeferencing

Licensing and Contracting Issues in Databases
LOC METS

Resource Identifier

Searching Digital Libraries

Data Models

Section Editor: David W. Embley

Abstraction

Conceptual Modeling Foundations: The Notion of a Model in Conceptual Modeling

Entity Relationship Model

Extended Entity-Relationship Model

Hierarchical Data Model

Key

Network Data Model

Object Constraint Language

Object Data Models

Object Identity

Object-Role Modeling

Relational Model

Semantic Data Model

Specialization and Generalization

Unified Modeling Language

\section{Complex Event Processing}

Section Editor: Opher Etzion

Complex Event Processing

Complex Event

Context

Event Causality

Event Channel

Event Cloud

Event Driven Architecture

Event Flow

Event Lineage

Event Pattern Detection

Event Prediction

Event Processing Agent

Event Processing Network

Event Sink

Event Source

Event Stream

Event Transformation

Event-Driven Business Process Management

Retrospective Event Processing

Uncertainty in Events 
Database Security and Privacy

Section Editor: Elena Ferrari

Access Control Administration Policies

Access Control Policy Languages

Access Control

Administration Model for RBAC

Anonymity

ANSI/INCITS RBAC Standard

Asymmetric Encryption

Auditing and Forensic Analysis

Authentication

Blind Signatures

Data Encryption

Data Rank/Swapping

Database Security

Digital Rights Management

Digital Signatures

Disclosure Risk

Discretionary Access Control

GEO-RBAC Model

Hash Functions

Homomorphic Encryption

Inference Control in Statistical

Databases

Information Loss Measures

Intrusion Detection Technology

$k$-Anonymity

Mandatory Access Control

Merkle Trees

Message Authentication Codes

Microaggregation

Microdata Rounding

Microdata

Multilevel Secure Database Management System

Noise Addition

Nonperturbative Masking Methods

PRAM

Privacy Policies and Preferences

Privacy Through Accountability

Privacy-Enhancing Technologies

Privacy-Preserving DBMSs

Privacy

Private Information Retrieval

Protection from Insider Threats

Pseudonymity

Record Linkage
Regulatory Compliance in Data

Management

Role-Based Access Control

SDC Score

Secure Data Outsourcing

Secure Database Development

Secure Transaction Processing

Security Services

Steganography

Symmetric Encryption

Synthetic Microdata

Tabular Data

Trusted Hardware

Unobservability

\section{Semantic Web and Ontologies}

Section Editor: Avigdor Gal

Description Logics

Emergent Semantics

Integration of Rules and Ontologies

Lightweight Ontologies

Linked Open Data

Ontology Elicitation

Ontology Engineering

Ontology

OWL: Web Ontology Language

Resource Description Framework (RDF)

Schema (RDFS)

Semantic Crowdsourcing

Semantic Matching

Semantic Social Web

Semantic Streams

Semantic Web Query Languages

Semantic Web Services

Semantic Web

\section{Data Cleaning}

\section{Section Editor: Venkatesh Ganti}

Column Segmentation

Constraint-Driven Database Repair

Data Cleaning

Data Profiling

Deduplication in Data Cleaning

Record Matching 


\section{Web Data Extraction}

\section{Section Editor: Georg Gottlob}

Data Integration in Web Data Extraction System

Fully Automatic Web Data Extraction

GUIs for Web Data Extraction

Languages for Web Data Extraction

Logical Foundations of Web Data Extraction

Screen Scraper

Snippet

Web Data Extraction System

Web ETL

Web Harvesting

Wrapper Induction

Wrapper Maintenance

Wrapper Stability

\section{Sensor Networks}

\section{Section Editor: Le Gruenwald}

Applications of Sensor Network Data

Management

Continuous Queries in Sensor Networks

Data Acquisition and Dissemination in

Sensor Networks

Data Aggregation in Sensor Networks

Data Compression in Sensor Networks

Data Estimation in Sensor Networks

Data Fusion in Sensor Networks

Data Storage and Indexing in Sensor Networks

Data Uncertainty Management in Sensor

Networks

Database Languages for Sensor Networks

In-Network Query Processing

Mobile Sensor Network Data Management

Model-Based Querying in Sensor Networks

Query Optimization in Sensor Networks

Sensor Networks

\section{Data Clustering}

\section{Section Editor: Dimitrios Gunopulos}

Cluster and Distance Measure

Clustering Overview and Applications

Clustering Validity
Clustering with Constraints

Density-Based Clustering

Dimension Reduction Techniques for

Clustering

Dimensionality Reduction Techniques for

Nearest-Neighbor Computations

Document Clustering

Feature Selection for Clustering

Hierarchical Clustering

Indexing and Similarity Search

Learning Distance Measures

Outlier Detection

Semi-supervised Learning

Spectral Clustering

Subspace Clustering Techniques

Visualizing Clustering Results

\section{Scientific Databases}

\section{Section Editor: Amarnath Gupta}

Annotation

Archiving Experimental Data

Array Databases

Biological Networks

Biological Sequences

Bitmap-Based Index Structures

Data Provenance

Data Types in Scientific Data Management

Database Techniques to Improve Scientific

Simulations

Database Use in Science Applications

Geo Raster Data Management

Graph Data Management in Scientific

Applications

Information Integration Techniques for Scientific

Data

Mining of Chemical Data

Multidimensional Data Formats

Query Evaluation Techniques for

Multidimensional Data

Sampling Techniques for Statistical Databases

Scientific Databases

Statistical Data Management

Storage of Large Scale Multidimensional

Data

Uncertainty Management in Scientific Database

Systems 


\section{Geographical Information Systems}

\section{Section Editor: Ralf Hartmut Güting}

Cardinal Direction Relationships

Crowdsourcing Geographic Information Systems

Digital Elevation Models

Dimension-Extended Topological Relationships

Field-Based Spatial Modeling

Geographic Information System

Geographical Information Retrieval

Geography Markup Language

Multi-resolution Terrain Modeling

Multiple Representation Modeling

Semantic Modeling for Geographic Information

Systems

Simplicial Complex

Spatial Data Analysis

Spatial Data Types

Spatial Network Databases

Spatial Operations and Map Operations

Three-Dimensional GIS and Geological

$$
\text { Applications }
$$

Time Aggregated Graphs

Topological Data Models

Topological Relationships

Triangulated Irregular Network

\section{Data Visualization}

\section{Section Editor: Hans Hinterberger}

Chart

Comparative Visualization

Data Visualization

Dynamic Graphics

Exploratory Data Analysis

Graph

Multivariate Visualization Methods

Parallel Coordinates

Scientific Visualization

Symbolic Representation

Table

Thematic Map

Visualizing Categorical Data

Visualizing Hierarchical Data
Visualizing Network Data

Visualizing Quantitative Data

\section{Web Services and Service Oriented Architectures}

\section{Section Editor: Hans-Arno Jacobsen}

\section{AJAX}

Business Process Execution Language

Business Process Management

Business Process Modeling Notation

Channel-Based Publish/Subscribe

Choreography

Composition

Content-Based Publish/Subscribe

Coordination

CORBA

Coupling and Decoupling

Database Adapter and Connector

DCE

DCOM

Discovery

.NET Remoting

Enterprise Application Integration

Enterprise Service Bus

Interface

Java Database Connectivity

Loose Coupling

MashUp

OASIS

Open Database Connectivity

Orchestration

Publish/Subscribe

Request Broker

RMI

Service Component Architecture (SCA)

Service-Oriented Architecture

SOAP

State-Based Publish/Subscribe

Subject Spaces

Tight Coupling

Topic-Based Publish/Subscribe

Type-Based Publish/Subscribe

W3C

Web 2.0/3.0

Web Services 


\section{Metadata Management}

\section{Section Editor: Manfred Jeusfeld}

Common Warehouse Metamodel

Data Dictionary

Data Warehouse Metadata

Deep Instantiation

Dublin Core

Geospatial Metadata

Java Metadata Facility

Meta Data Repository

Meta Object Facility

Metadata Interchange Specification

Metadata Registry, ISO/IEC 11179

Metadata

Metamodel

Model Management

Multilevel Modeling

Resource Description Framework

Stream Reasoning

Telos

Topic Maps

XML Metadata Interchange

\section{Health Informatics Databases}

\section{Section Editor: Vipul Kashyap}

Biomedical Data/Content Acquisition, Curation

Biomedical Image Data Types and Processing

Biomedical Scientific Textual Data Types and Processing

Clinical Data Acquisition, Storage, and Management

Clinical Data and Information Models

Clinical Data Quality and Validation

Clinical Decision Support

Clinical Document Architecture

Clinical Event

Clinical Knowledge Repository

Clinical Observation

Clinical Ontologies

Clinical Order

Computerized Physician Order Entry

Data Privacy and Patient Consent

Data Warehousing for Clinical Research
Data, Text, and Web Mining in Healthcare

Electronic Health Record

Enterprise Terminology Services

Evidence-Based Medicine

Executable Knowledge

Implications of Genomics for Clinical

Informatics

Interface Engines in Healthcare

Interoperation of NLP-Based Systems with Clinical Databases

Quality and Trust of Information Content and Credentialing

Reference Knowledge

Taxonomy: Biomedical Health Informatics

Workflow Management Systems

\section{Visual Data Mining}

Section Editor: Daniel A. Keim

Dense Pixel Displays

Distortion Techniques

Iconic Displays

KDD Pipeline

Linking and Brushing

Treemaps

Visual Analytics

Visual Association Rules

Visual Classification

Visual Clustering

Visual Data Mining

Visualization Pipeline

Zooming Techniques

\section{Data Replication}

\section{Section Editor: Bettina Kemme}

Autonomous Replication

Causal Consistency

Concurrency Control for Replicated Databases

Consistency Models for Replicated Data

Data Replication

Eventual Consistency

One-Copy-Serializability

Optimistic Replication and Resolution

Partial Replication

Quorum Systems 
Replica Control

Replica Freshness

Replicated Data Types

Replication Based on Group Communication

Replication Based on Paxos

Replication for Availability and Fault Tolerance

Replication for Scalability

Strong Consistency Models for Replicated Data

WAN Data Replication

Weak Consistency Models for Replicated Data

\section{Storage Structures and Systems}

\section{Section Editor: Masaru Kitsuregawa}

Active Storage

Backup and Restore

Checksum and Cyclic Redundancy Check

Mechanism

Continuous Data Protection

Database Machine

Deduplication

Direct Attached Storage

Disaster Recovery

Disk Power Saving

Information Lifecycle Management

Intelligent Storage Systems

IP Storage

Logical Volume Manager

Massive Array of Idle Disks

Multitier Storage Systems

Network Attached Secure Device

Network Attached Storage

Point-in-Time Copy

Redundant Arrays of Independent Disks

Replication

SAN File System

Storage Area Network

Storage Consolidation

Storage Management Initiative Specification

Storage Management

Storage Network Architectures

Storage Networking Industry Association

Storage Power Management

Storage Protection

Storage Resource Management

Storage Virtualization

Write Once Read Many

\section{Views and View Management}

\section{Section Editor: Yannis Kotidis}

Answering Queries Using Views

Incremental Maintenance of Views with

Aggregates

Maintenance of Materialized Views with

Outer-Joins

Maintenance of Recursive Views

Self-Maintenance of Views

Side-Effect-Free View Updates

Updates Through Views

View Adaptation

View Definition

View Maintenance Aspects

View Maintenance

Views

Web Views

\section{Structured Text Retrieval}

Section Editor: Jaap Kamps

Aggregation-Based Structured Text Retrieval

Content-and-Structure Query

Content-Only Query

Contextualization in Structured Text Retrieval

Entity Retrieval

Evaluation Metrics for Structured Text Retrieval

Indexing Units of Structured Text Retrieval

Initiative for the Evaluation of XML Retrieval

Integrated DB and IR Approaches

Logical Document Structure

Managing Compressed Structured Text

Narrowed Extended XPath I

Presenting Structured Text Retrieval Results

Processing Overlaps in Structured Text Retrieval

Processing Structural Constraints

Profiles and Context for Structured Text

Retrieval

Propagation-Based Structured Text Retrieval

Relationships in Structured Text Retrieval

Relevance

Specificity

Structure Weight

Structured Document Retrieval

Structured Text Retrieval Models

Term Statistics for Structured Text Retrieval

XML Retrieval 


\section{Information Quality}

Section Editor: Yang W. Lee

Information Quality and Decision-Making

Information Quality Assessment

Information Quality Policy and Strategy

Information Quality: Managing Information

as a Product

\section{Relational Theory}

\section{Section Editor: Leonid Libkin}

Aggregation: Expressiveness and Containment Armstrong Axioms

Boyce-Codd Normal Form

BP-Completeness

Cartesian Product

Certain (and Possible) Answers

Chase

Computationally Complete Relational Query Languages

Conditional Tables

Constraint Databases

Consistent Query Answering

Constraint Query Languages

Database Dependencies

Database Repair

Difference

Ehrenfeucht-Fraïssé Games

Equality-Generating Dependencies

Expressive Power of Query Languages

Fourth Normal Form

Functional Dependency

Implication of Constraints

Incomplete Information

Inconsistent Databases

Incremental Computation of Queries

Join Dependency

Join

Locality of Queries

Membership Query

Multivalued Dependency

Naive Tables

Normal Forms and Normalization

Null Values

Order Dependency

Parameterized Complexity of Queries

Positive Relational Algebra
Possible Answers

Projection

Query Containment

Safety and Domain Independence

Second Normal Form (2NF)

Selection

Third Normal Form

Tuple-Generating Dependencies

Union

Zero-One Laws

Information Retrieval Evaluation Measures

Section Editor: Weiyi Meng

$\alpha-\mathrm{nDCG}$

Advanced Information Retrieval Measures

Average Precision at $\mathrm{n}$

Average Precision Histogram

Average Precision

Average R-Precision

Bpref

D-Measure

Discounted Cumulated Gain

Effectiveness Involving Multiple Queries

Eleven Point Precision-Recall Curve

ERR-IA

Expected Reciprocal Rank

F-Measure

GMAP

MAP

Mean Reciprocal Rank

Precision at $\mathrm{n}$

Precision-Oriented Effectiveness Measures

Precision

Q-Measure

R-Precision

Recall

Standard Effectiveness Measures

Success at $\mathrm{n}$

U-Measure

\section{Logical Data Integration}

Section Editor: Renée J. Miller

Data Exchange

Schema Mapping Composition

Schema Mapping

Schema Matching 


\section{Database Design}

\section{Section Editor: Alexander Borgida}

Conceptual Schema Design

Database Design

Database Reverse Engineering

Design for Data Quality

Logical Database Design: From Conceptual to Logical Schema

Physical Database Design for Relational

Databases

\section{Text Indexing Techniques}

\section{Section Editor: Mario A. Nascimento}

Indexing Compressed Text

Indexing the Web

Inverted Files

Signature Files

Suffix Tree

Text Compression

Text Indexing Techniques

Trie

\section{Data Quality}

\section{Section Editor: Felix Naumann}

Data Conflicts

Data Fusion

Data Quality Assessment

Data Quality Dimensions

Data Quality Models

Data Scrubbing

Probabilistic Databases

\section{Web Search and Crawl}

\section{Section Editor: Cong $\mathrm{Yu}$}

Deep-Web Search

Faceted Search

Focused Web Crawling

Geo-Targeted Web Search

Incremental Crawling

Metasearch Engines

Peer-to-Peer Web Search

Personalized Web Search

Precision and Recall

Search Engine Metrics
Test Collection

Web Advertising

Web Characteristics and Evolution

Web Crawler Architecture

Web Information Extraction

Web Page Quality Metrics

Web Question Answering

Web Search Query Rewriting

Web Search Relevance Feedback

Web Search Relevance Ranking

Web Search Result Caching and Prefetching

Web Search Result De-duplication and

Clustering

Web Spam Detection

\section{Multimedia Databases}

\section{Section Editor: Vincent Oria, Shin'ichi Satoh}

Audio Classification

Audio Content Analysis

Audio Metadata

Audio Representation

Audio Segmentation

Audio

Automatic Image Annotation

Computational Media Aesthetics

Content-Based Video Retrieval

Human-Centered Computing: Application to Multimedia

Image Content Modeling

Image Database

Image Metadata

Image Querying

Image Representation

Image Retrieval and Relevance Feedback

Image Segmentation

Image Similarity

Image

Multimedia Data Indexing

Multimedia Data Querying

Multimedia Databases

Multimedia Data

Multimedia Metadata

Object Recognition

Semantic Modeling and Knowledge

Representation for Multimedia Data

Video Content Analysis 
Video Content Modeling

Video Content Structure

Video Metadata

Video Querying

Video Representation

Video Scene and Event Detection

Video Shot Detection

Video Summarization

Video

Visual Content Analysis

\section{Active Databases}

\section{Section Editor: M. Tamer $\ddot{O}_{z s u}$}

Active Database Coupling Modes

Active Database Execution Model

Active Database Knowledge Model

Active Database Management System

Architecture

Active Database Rulebase

Active Database, Active Database (Management)

System

Atomic Event

Composite Event

Database Trigger

ECA Rule Action

ECA Rule Condition

ECA Rules

Event Detection

Event in Active Databases

Event Specification

Explicit Event

Implicit Event

Spatial, Spatiotemporal, and

Multidimensional Databases

\section{Section Editor: Dimitris Papadias}

Air Indexes for Spatial Databases

Closest-Pair Query

Continuous Monitoring of Spatial

Queries

Curse of Dimensionality

Distributed Spatial Databases

Geo-Social Networks

Grid File (and Family)
High-Dimensional Indexing

Indexing Historical Spatiotemporal Data

Indexing Metric Spaces

Indexing of the Current and Near-Future

Positions of Moving Objects

Metric Space

Multi step Query Processing

Nearest Neighbor Query in Spatiotemporal

Databases

Nearest Neighbor Query

Probabilistic Spatial Queries

Quadtrees (and Family)

R-Tree (and Family)

Resource Allocation Problems in Spatial

Databases

Reverse Nearest Neighbor Query

Road Networks

Space-Filling Curves for Query

Processing

Space-Filling Curves

Spatial and Spatiotemporal Data Models and

Languages

Spatial Anonymity

Spatial Data Mining

Spatial Indexing Techniques

Spatial Join

Spatial Matching Problems

Spatial Queries in the Cloud

Spatial-Keyword Search

Spatiotemporal Data Mining

Spatiotemporal Data Warehouses

Spatiotemporal Interpolation Algorithms

Spatiotemporal Selectivity Estimation

Spatiotemporal Trajectories

Trip Planning Queries

Voronoi Diagrams

\section{Data Warehouse}

\section{Section Editor: Torben Bach Pedersen,} Stefano Rizzi

Active, Real-Time, and Intellective Data

Warehousing

Business Intelligence

Cloud Intelligence

Cube Implementations

Cube 
Data Mart

Data Warehouse Life Cycle and Design

Data Warehouse Maintenance, Evolution, and Versioning

Data Warehouse Security

Data Warehouse

Data Warehousing in Cloud Environments

Data Warehousing on Nonconventional Data

Data Warehousing Systems: Foundations and Architectures

Dimension

Extraction, Transformation, and Loading

Hierarchy

Indexing of Data Warehouses

Interoperability in Data Warehouses

Join Index

Measure

Multidimensional Modeling

OLAM

OLAP Personalization and Recommendation

Online Analytical Processing

Optimization and Tuning in Data

Warehouses

Parallel and Distributed Data Warehouses

Predictive Analytics

Prescriptive Analytics

Quality of Data Warehouses

Query Processing in Data Warehouses

Snowflake Schema

Spatial Datawarehousing

Star Index

Star Schema

Summarizability

Temporal Datawarehousing

Visual Online Analytical Processing

(OLAP)

What-If Analysis

\section{Association Rule Mining}

\section{Section Editor: Jian Pei}

Anti-monotone Constraints

Applications of Emerging Patterns for Microarray Gene Expression Data Analysis

Approximation of Frequent Itemsets Apriori Property and Breadth-First Search

Algorithms
Association Rules

Closed Itemset Mining and Nonredundant Association Rule Mining

Convertible Constraints

Data Mining

Emerging Pattern Based Classification

Emerging Patterns

Frequent Graph Patterns

Frequent Itemset Mining with Constraints

Frequent Itemsets and Association Rules

Frequent Partial Orders

Max-Pattern Mining

Monotone Constraints

Pattern-Growth Methods

Quantitative Association Rules

Sequential Patterns

Succinct Constraints

\section{Workflow Management}

Section Editor: Barbara Pernici

Activity Diagrams

Activity

Actors/Agents/Roles

Business Process Modeling

Business Process Reengineering

Control Data

Grid and Workflows

Loop

OR-Join

OR-Split

Petri Nets

Process Life Cycle

Process Mining

Process Optimization

Scheduler

Scientific Workflows

Split

Workflow Constructs

Workflow Evolution

Workflow Join

Workflow Management and Workflow

Management System

Workflow Management Coalition

Workflow Management

Workflow Model Analysis

Workflow Model 
Workflow Patterns

Workflow Schema

XML Process Definition Language

Query Processing and Optimization

Section Editor: Evaggelia Pitoura

Access Path

Adaptive Query Processing

Buffer Management

Cost Estimation

Evaluation of Relational Operators

Hash Join

Index Join

Iterator

Join Order

Multi-query Optimization

Nested Loop Join

Pipelining

Query Optimization (in Relational

Databases)

Query Optimization

Query Plan

Query Processing (in Relational

Databases)

Query Processing and Optimization in Object

Relational Databases

Query Processing in Deductive Databases

Query Processing

Query Rewriting

Selectivity Estimation

Sort-Merge Join

System R (R*) Optimizer

\section{Data Management for the Life Sciences}

\section{Section Editor: Louiqa Raschid}

Biological Metadata Management

Biological Resource Discovery

Biostatistics and Data Analysis

Data Integration Architectures and Methodology for the Life Sciences

Gene Expression Arrays

Graph Management in the Life Sciences

Image Management for Biological Data

Index Structures for Biological Sequences
Machine Learning in Computational Biology

Ontologies and Life Science Data Management

Query Languages and Evaluation Techniques

for Biological Sequence Data

Query Languages for the Life Sciences

Semantic Data Integration for Life Science Entities

Text Mining of Biological Resources

Web Services and the Semantic Web for Life

Science Data

\section{Information Retrieval Operations}

\section{Section Editor: Edie Rasmussen}

Clustering for Post Hoc Information

Retrieval

Index Creation and File Structures

Information Filtering

Information Retrieval Operations

Lexical Analysis of Textual Data

Query Expansion for Information

Retrieval

Relevance Feedback for Text Retrieval

Similarity and Ranking Operations

Stemming

Stoplists

Summarization

Term Weighting

Text Index Compression

TF*IDF

Visualization for Information Retrieval

\section{Query Languages}

Section Editor: Tore Risch

AMOSQL

Comprehensions

Daplex

FQL

Functional Data Model

Functional Query Language

OQL

OSQL

P/FDM

QUEL

Query Language 
SQL

Stack-Based Query Language

Stored Procedure

\section{Database Tuning and Performance}

\section{Section Editor: Dennis Shasha}

Application-Level Tuning

Benchmark Frameworks

Data Generation

Database Benchmarks

Index Tuning

Physical Layer Tuning

Schema Tuning

Tuning Concurrency Control

Classification and Decision Trees

\section{Section Editor: Kyuseok Shim}

Approximate Reasoning

Bagging

Bayesian Classification

Boosting

Bootstrap

Classification by Association Rule

$$
\text { Analysis }
$$

Classification

Cross-Validation

Decision Rule Mining in Rough Set Theory

Decision Tree Classification

Decision Trees

Deductive Data Mining Using Granular

$$
\text { Computing }
$$

Ensemble

Fuzzy Relation

Fuzzy Set

Fuzzy Set Approach

Fuzzy/Linguistic IF-THEN Rules and Linguistic Descriptions

Genetic Algorithms

Nearest Neighbor Classification

Neural Networks

Receiver Operating Characteristic

Residuated Lattice

Rule-Based Classification

Scalable Decision Tree Construction
Support Vector Machine

Triangular Norms

\section{Temporal Databases}

Section Editor: Christian S. Jensen, Richard T. Snodgrass

Absolute Time

Abstract Versus Concrete Temporal Query Languages

Allen's Relations

Applicability Period

Atelic Data

Bitemporal Indexing

Bitemporal Interval

Bitemporal Relation

Calendar

Calendric System

Chronon

Current Semantics

Event in Temporal Databases

Fixed Time Span

Forever

History in Temporal Databases

Lifespan

Nonsequenced Semantics

Now in Temporal Databases

Period-Stamped Temporal Models

Physical Clock

Point-Stamped Temporal Models

Probabilistic Temporal Databases

Qualitative Temporal Reasoning

Relative Time

Schema Evolution

Schema Versioning

Sequenced Semantics

Snapshot Equivalence

SQL-Based Temporal Query Languages

Supporting Transaction Time Databases

tBench

Telic Distinction in Temporal Databases

Temporal Access Control

Temporal Aggregation

Temporal Algebras

Temporal Benchmarks

Temporal Coalescing

Temporal Compatibility 
Temporal Conceptual Models

Temporal Constraints

Temporal Data Mining

Temporal Data Models

Temporal Database

Temporal Dependencies

Temporal Element

Temporal Expression

Temporal Extensions in the SQL

Standard

Temporal Generalization

Temporal Granularity

Temporal Homogeneity

Temporal Indeterminacy

Temporal Integrity Constraints

Temporal Joins

Temporal Logic in Database Query

Languages

Temporal Logical Models

Temporal Object-Oriented Databases

Temporal Periodicity

Temporal Projection

Temporal PSM

Temporal Query Languages

Temporal Query Processing

Temporal Relational Calculus

Temporal Specialization

Temporal Strata

Temporal Vacuuming

Temporal XML

Time Domain

Time in Philosophical Logic

Time Instant

Time Interval

Time Period

Time Series Query

Time Span

Time-Line Clock

Timeslice Operator

Transaction Time

Transaction-Time Indexing

TSQL2

User-Defined Time

Valid Time

Valid-Time Indexing

Value Equivalence

Variable Time Span

Weak Equivalence

\section{Stream Mining}

\section{Section Editor: Divesh Srivastava}

AMS Sketch

Anomaly Detection on Streams

Association Rule Mining on Streams

Change Detection on Streams

Classification in Streams

Clustering on Streams

Count-Min Sketch

Decay Models

FM Synopsis

Frequency Moments

Frequent Items on Streams

Geometric Stream Mining

Graph Mining on Streams

Hierarchical Heavy Hitter Mining on

Streams

Histograms on Streams

Quantiles on Streams

Stable Distribution

Stream Mining

Stream Models

Stream Sampling

Stream Similarity Mining

Synopsis Structure

Wavelets on Streams

\section{Distributed Database Systems}

Section Editor: Kian-Lee Tan

Distributed Architecture

Distributed Database Design

Distributed Database Systems

Distributed Deadlock Management

Distributed Join

Distributed Query Optimization

Distributed Query Processing

Distributed Recovery

Distributed Transaction Management

Graph OLAP

Indexing in Pub/Sub Systems

Semijoin Program

Semijoin

Three-Phase Commit

Two-Phase Commit

Window Operator in RDBMS 


\section{Logics and Databases}

\section{Section Editor: Val Tannen}

Bag Semantics

Conjunctive Query

Datalog

First-Order Logic: Semantics

First-Order Logic: Syntax

FOL Modeling of Integrity Constraints

(Dependencies)

Relational Algebra

Relational Calculus

\section{Structured and Semi-structured Document} Databases

\section{Section Editor: Frank Tompa}

Document Databases

Document Representations (Inclusive Native and Relational)

Electronic Dictionary

Electronic Encyclopedia

Electronic Newspapers

Enterprise Content Management

Functional Dependencies for Semistructured

$$
\text { Data }
$$

Grammar Inference

Hypertexts

Markup Language

Normal Form ORA-SS Schema Diagrams

Object Relationship Attribute Data Model for Semistructured Data

Path Query

Region Algebra

Semi-structured Data Model

Semi-structured Database Design

Semi-structured Query Languages

Structural Indexing

Unicode

Indexing

Section Editor: Vassilis J. Tsotras

B+-Tree

Bitmap Index

Bloom Filters
Covering Index

Dense Index

Extendible Hashing

Generalized Search Tree

Hash-Based Indexing

I/O Model of Computation

Indexed Sequential Access Method

Linear Hashing

Primary Index

Range Query

Secondary Index

Sparse Index

Tree-Based Indexing

\section{Parallel Database Systems}

\section{Section Editor: Patrick Valduriez.}

Data Skew

Database Clusters

Inter-operator Parallelism

Intra-operator Parallelism

Online Recovery in Parallel Database Systems

Parallel Data Placement

Parallel Database Management

Parallel Hash Join, Parallel Merge Join, Parallel

Nested Loops Join

Parallel Query Execution Algorithms

Parallel Query Optimization

Parallel Query Processing

Query Load Balancing in Parallel Database

Systems

Shared-Disk Architecture

Shared-Memory Architecture

Shared-Nothing Architecture

Virtual Partitioning

\section{Advanced Storage Systems}

\section{Section Editor: Kaladhar Voruganti}

Initiator

Logical Unit Number Mapping

Logical Unit Number

Multi-pathing

Object Storage Protocol

SCSI Target

Software-Defined Storage 
Solid State Drive (SSD)

Storage Access Models

Storage Devices

Storage Grid

Storage Protocols

Storage Security

Volume

\section{Transaction Management}

\section{Section Editor: Gottfried Vossen}

ACID Properties

Application Recovery

Atomicity

B-Tree Locking

Concurrency Control: Traditional Approaches

Crash Recovery

Distributed Concurrency Control

Escrow Transactions

Locking Granularity and Lock Types

Logging and Recovery

Multilevel Recovery and the ARIES Algorithm

Multiversion Serializability and Concurrency

Control

Performance Analysis of Transaction Processing

Systems

Replicated Database Concurrency Control

Replication with Snapshot Isolation

Serializability

Serializable Snapshot Isolation

Snapshot Isolation

Software Transactional Memory

SQL Isolation Levels

Transaction Chopping

Transaction Management

Transaction Models: The Read/Write Approach

Transaction

Two-Phase Commit Protocol

Two-Phase Locking

Mobile and Ubiquitous Data Management

\section{Section Editor: Ouri Wolfson}

Compression of Mobile Location Data

Data Broadcasting, Caching, and Replication in Mobile Computing
Data Management for VANETs

Location Management in Mobile

Environments

Location-Based Recommendation

Location-Based Services

MANET Databases

Map Matching

Mobile Database

Mobile Resource Search

Moving Objects Databases and Tracking

Moving Object

Real and Synthetic Test Datasets

Spatiotemporal Data Types

Trajectory

\section{Multimedia Information Retrieval}

\section{Section Editor: Jeffrey $\mathrm{Xu} \mathrm{Yu}$}

Annotation-Based Image Retrieval

Continuous Multimedia Data Retrieval

Cross-Modal Multimedia Information

\section{Retrieval}

Electronic Ink Indexing

Feature Extraction for Content-Based Image

Retrieval

Feature-Based 3D Object Retrieval

Indexing Techniques for Multimedia Data

Retrieval

Multimedia Data Buffering

Multimedia Data Storage

Multimedia Information Retrieval Model

Multimedia Resource Scheduling

Multimedia Retrieval Evaluation

Multimedia Tagging

Near-Duplicate Retrieval

Query by Humming

Query Point Movement Techniques for

Content-Based Image Retrieval

Relevance Feedback for Content-Based

Information Retrieval

Social Media Harvesting

Top-K Selection Queries on Multimedia

Datasets

Video Segmentation

Video Sequence Indexing 
Approximation and Data Reduction

Techniques

\section{Section Editor: Xiaofang Zhou}

Approximate Query Processing

Data Reduction

Data Sampling

Data Sketch/Synopsis

Database Clustering Methods

Dimensionality Reduction

Discrete Wavelet Transform and Wavelet Synopses

Fractal

Hierarchical Data Summarization

Histogram

K-Means and K-Medoids

Linear Regression

Log-Linear Regression

Multidimensional Scaling

Nonparametric Data Reduction Techniques

Parametric Data Reduction Techniques

Principal Component Analysis

Singular Value Decomposition

Two-Dimensional Shape Retrieval

\section{Social Networks}

\section{Section Editor: Nick Koudas}

Collaborative Filtering

Recommender Systems

Social Influence

Social Media Analysis

\section{Cloud Data Management}

\section{Section Editor: Amr El Abbadi}

CAP Theorem

Cloud Computing

Data Center Energy Efficiency

Data Management in Data Centers

Data Migration Management

Elasticity

Infrastructure-as-a-Service (IaaS)

Multi-data Center Replication Protocols

Multi-datacenter Consistency Properties

Multitenancy
Platform-as-a-Service (PaaS)

Software-as-a-Service (SaaS)

Data Analytics

Section Editor: Fatma Özcan

Big Data Platforms for Data Analytics

Distributed File Systems

Distributed Machine Learning

Interactive Analytics in Social Media

Social Media Analytics

SQL Analytics on Big Data

Streaming Analytics

Structure Analytics in Social Media

Temporal Analytics in Social Media

Text Analytics in Social Media

Text Analytics

\section{Data Management Fundamentals}

Section Editor: Ramez Elmasri

Data Definition Language (DDL)

Data Definition

Data Management Fundamentals: Database

Management System

Data Manipulation Language (DML)

Database Administrator (DBA)

Database Schema

Database

Logical and Physical Data Independence

\section{NoSQL Databases}

Section Editor: Ling Liu and M. Tamer $\ddot{O}_{z s u}$

Column Stores

Document

MapReduce

NoSQL Stores

\section{Graph Data Management}

Section Editor: Lei Chen

Graph Database

Graph Mining 
RDF Stores

RDF Technology

SPARQL

\section{Data Provenance Management}

\section{Section Editor: Juliana Freire}

Provenance and Reproducibility

Provenance in Databases

Provenance in Scientific Databases

Provenance in Workflows

Provenance Standards

Provenance Storage

Provenance: Privacy and Security

\section{Ranking Queries}

\section{Section Editor: Ihab F. Ilyas}

Preference Queries

Preference Specification

Probabilistic Skylines

Rank-Aware Query Processing

Rank-Join Indices

Rank-Join

Ranking Views

Reverse Top-k Queries

Score Aggregation

Skyline Queries and Pareto Optimality

Top-k Queries

Uncertain Top-k Queries
Uncertain Data Management

Section Editor: Minos Garofalakis

Graphical Models for Uncertain Data Management

Indexing Uncertain Data

Karp-Luby Sampling

Managing Data Integration Uncertainty

Managing Probabilistic Entity Extraction

Monte Carlo Methods for Uncertain Data

Probabilistic Entity Resolution

Query Processing over Uncertain Data

Uncertain Data Lineage

Uncertain Data Mining

Uncertain Data Models

Uncertain Data Streams

Uncertain Graph Data Management

Uncertain Spatial Data Management

\section{Crowd Sourcing}

\section{Section Editor: Reynold Cheng}

Cost and Quality Trade-Offs in Crowdsourcing

Crowd Database Operators

Crowd Database Systems

Crowd Mining and Analysis

Human Factors Modeling in Crowdsourcing

Indexing with Crowds 


\section{About the Editors}

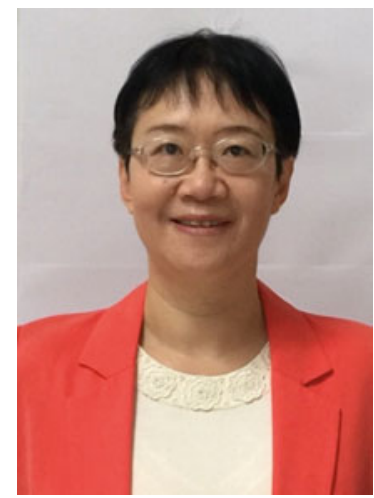

Ling Liu Georgia Institute of Technology College of Computing, Atlanta, GA, USA

Ling Liu is Professor of Computer Science in the College of Computing at Georgia Institute of Technology. She holds a Ph.D. (1992) in Computer and Information Science from Tilburg University, The Netherlands. Dr. Liu directs the research programs in the Distributed Data Intensive Systems Lab (DiSL), examining various aspects of data intensive systems, ranging from big data systems, cloud computing, databases, Internet and mobile systems and services, machine learning, to social and crowd computing, with the focus on performance, availability, security, privacy, and trust. Prof. Liu is an elected IEEE Fellow and a recipient of IEEE Computer Society Technical Achievement Award (2012). She has published over 300 international journal and conference articles and is a recipient of the best paper award from numerous top venues, including ICDCS, WWW, IEEE Cloud, IEEE ICWS, and ACM/IEEE CCGrid. In addition to serving as general chair and PC chairs of numerous IEEE and ACM conferences in big data, distributed computing, cloud computing, data engineering, and very large databases fields, Prof. Liu served as the Editor-in-Chief of IEEE Transactions on Service Computing (2013-2016) and also served on editorial boards of over a dozen international journals. Her current research is sponsored primarily by NSF and IBM. 


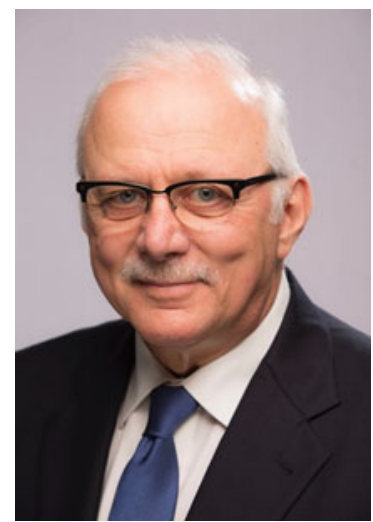

M. Tamer Özsu University of Waterloo School of Computer Science, Waterloo, ON, Canada

M. Tamer Özsu is Professor of Computer Science at the David R. Cheriton School of Computer Science and the Associate Dean of Research of the Faculty of Mathematics at the University of Waterloo. He was the Director of the Cheriton School of Computer Science from January 2007 to June 2010.

His research is in data management focusing on large-scale data distribution and management of nontraditional data, currently focusing on graph and RDF data. His publications include the book Principles of Distributed Database Systems (with Patrick Valduriez), which is now in its third edition. He was the Founding Series Editor of Synthesis Lectures on Data Management (Morgan \& Claypool) and is now the Editor-in-Chief of ACM Books. He serves on the editorial boards of three journals and two book series.

He is a Fellow of the Royal Society of Canada, American Association for the Advancement of Science (AAAS), Association for Computing Machinery (ACM), and the Institute of Electrical and Electronics Engineers (IEEE). He is an elected member of the Science Academy, Turkey, and a member of Sigma Xi. He was awarded the ACM SIGMOD Test-of-Time Award in 2015, the ACM SIGMOD Contributions Award in 2006, and the Ohio State University College of Engineering Distinguished Alumnus Award in 2008. 


\section{Advisory Board}

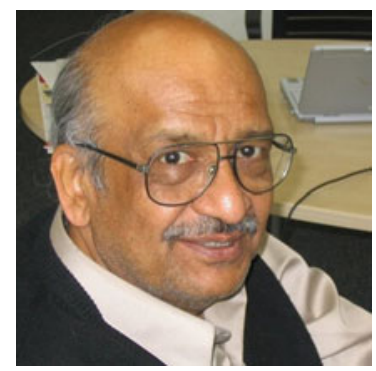

Ramesh Jain Department of Computer Science, School of Information and Computer Sciences, University of California Irvine, Irvine, CA, USA

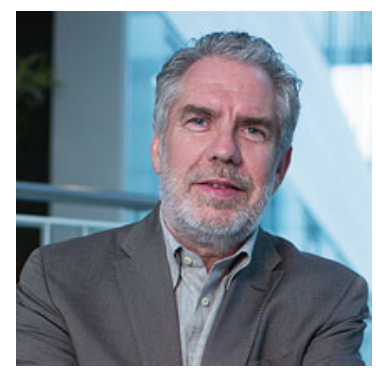

Peter MG Apers Centre for Telematics and Information Technology, University of Twente, Enschede, The Netherlands

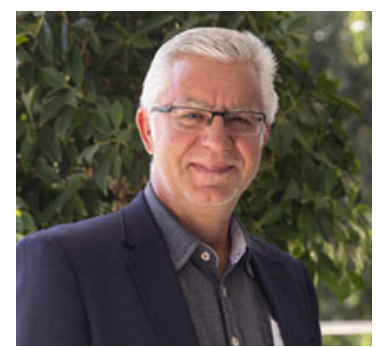

Timos Sellis Data Science Research Institute, Swinburne University of Technology, Hawthorn, VIC, Australia 


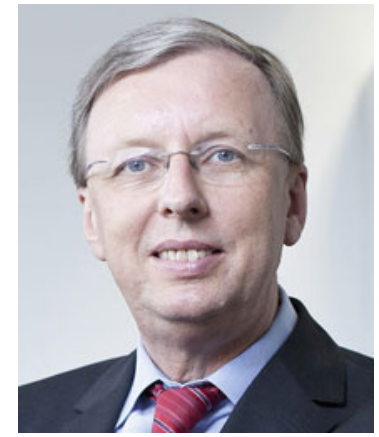

Matthias Jarke Informatik 5 Information Systems, RWTH-Aachen University, Aachen, Germany

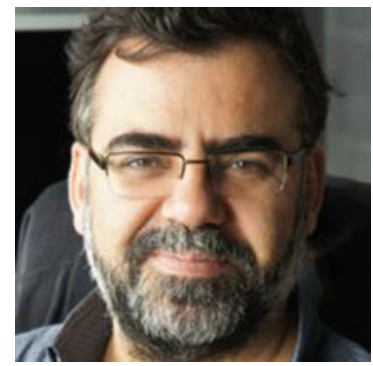

Ricardo Baeza-Yate Department of Information and Communication Technologies, University of Pompeu Fabra, Barcelona, Spain

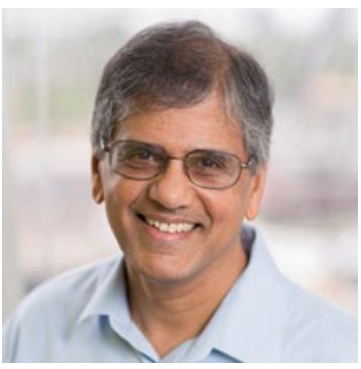

Jai Menon Cloudistics, Reston, VA, USA

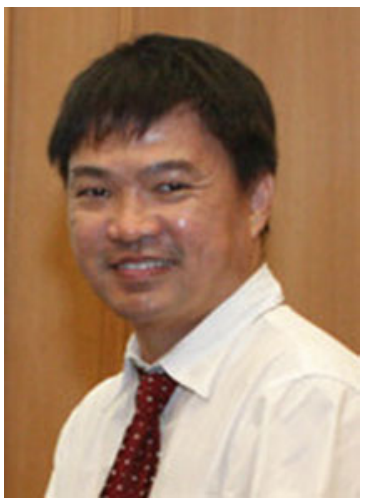

Beng Chin Ooi School of Computing, National University of Singapore, Singapore, Singapore 


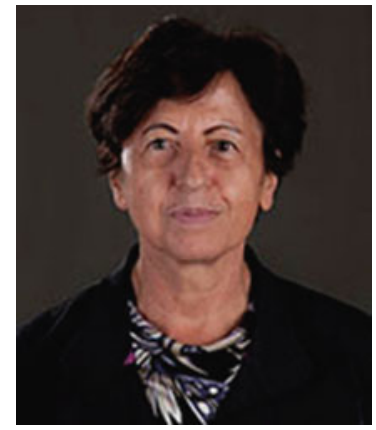

Elisa Bertino Department of Computer Science, Purdue University, West Lafeyette, IN, USA

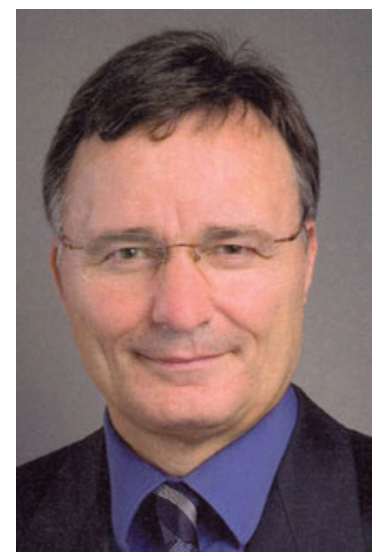

Erhard Rahm Fakultät für Mathematik und Informatik, Institut für Informatik, Universität Leipzig, Leipzig, Germany

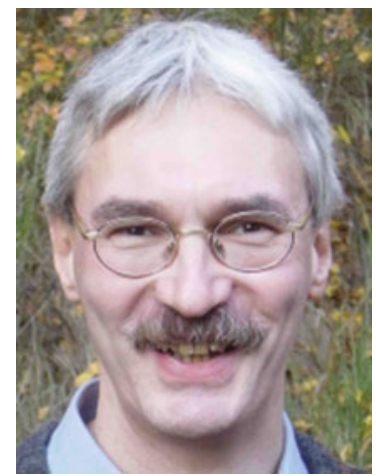

Gerhard Weikum Department 5: Databases and Information Systems, Max-Planck-Institut für Informatik, Saarbrücken, Germany 


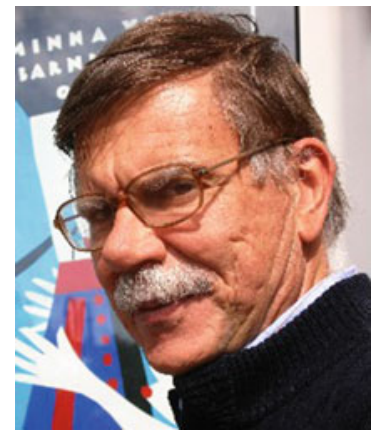

Stefano Ceri Department of Electronics, Information and Bioengineering, Politecnico di Milano, Milano, Italy

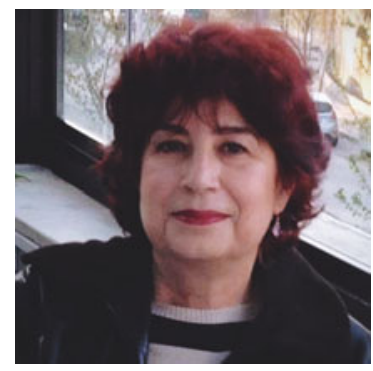

Asuman Dogac SRDC Software Research and Development and Consultancy Ltd., Cankaya/Ankara, Turkey

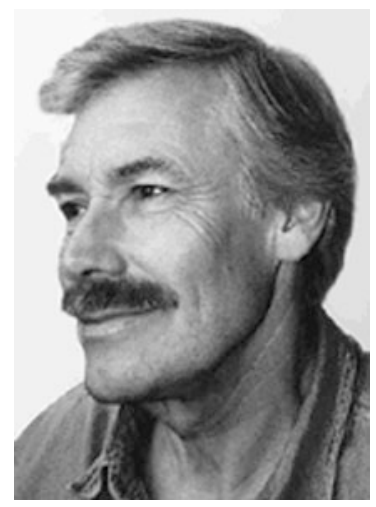

Hans-Joerg Schek Department of Computer Science, ETH Zürich, Zürich, Switzerland 


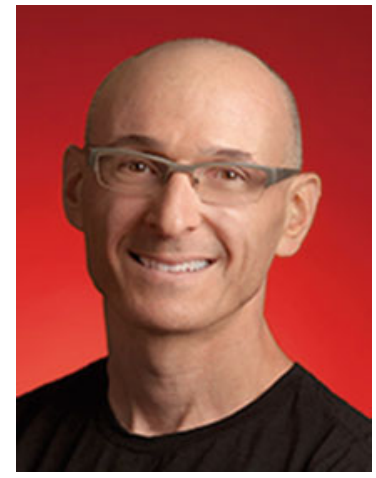

Alon Halevy Recruit Institute of Technology, Mountain View, CA, USA

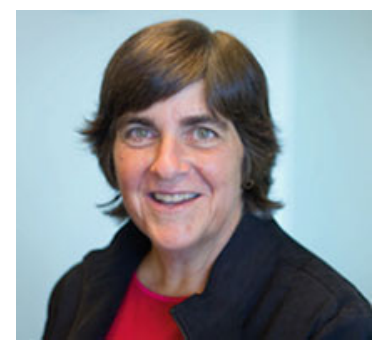

Jennifer Widom Frederick Emmons Terman School of Engineering, Stanford University, Stanford, CA, USA

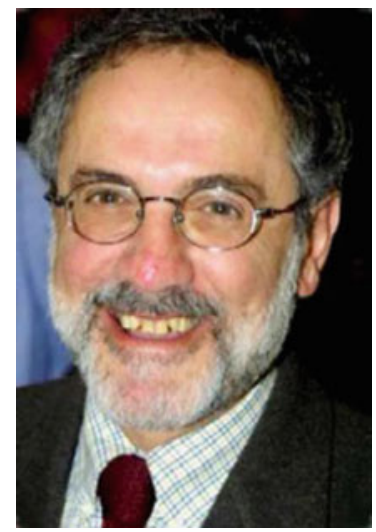

John Mylopoulos Department of Computer Science, University of Toronto, Toronto, ON, Canada 


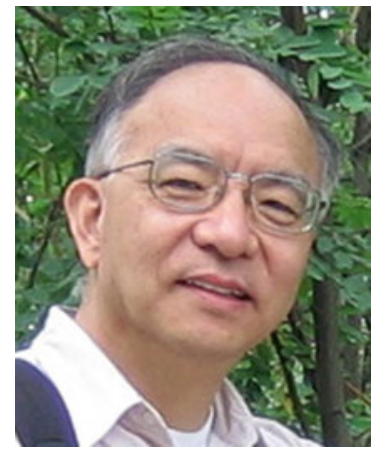

Jiawei Han Department of Computer Science, University of Illinois at Urbana-Champaign, Urbana, IL, USA

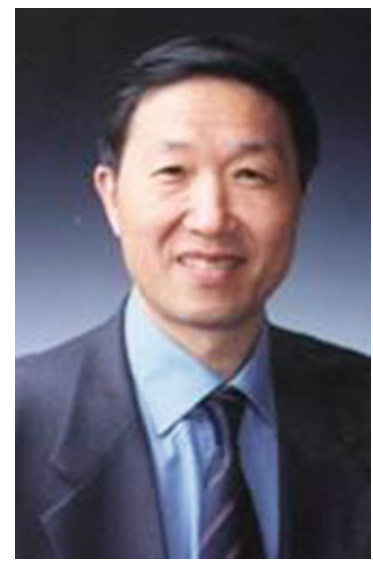

Lizhu Zhou Department of Computer Science and Technology, Tsinghua University, Beijing, China

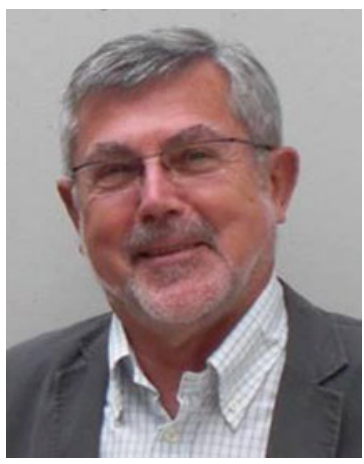

Theo Härder Department of Computer Science, University of Kaiserslautern, Kaiserslautern, Germany 


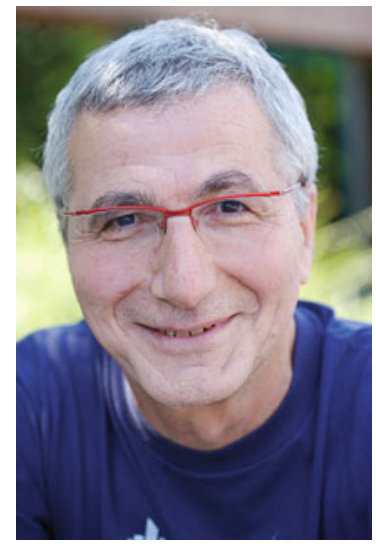

Serge Abiteboul INRIA and ENS, Paris, France

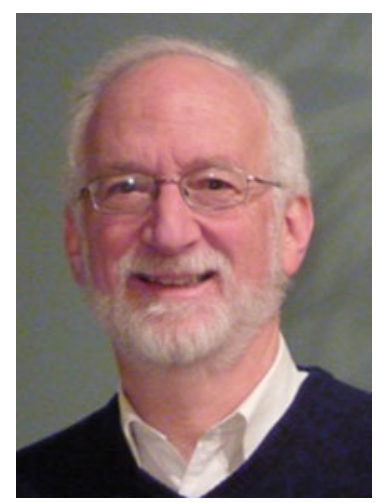

Frank Tompa David R. Cheriton School of Computer Science, University of Waterloo, Waterloo, ON, Canada

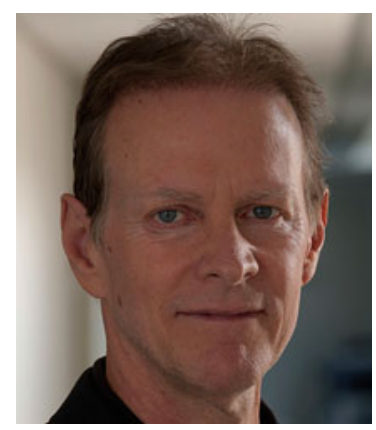

Patrick Valduriez INRIA and LIRMM, Montpellier, France 


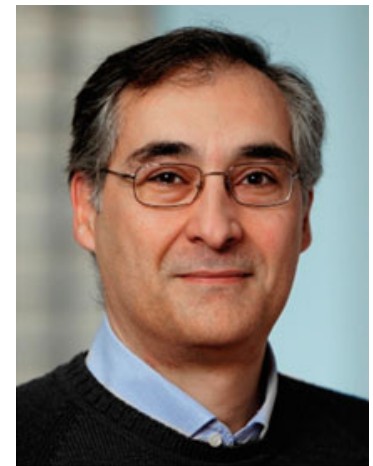

Gustavo Alonso Department of Computer Science, ETH Zürich, Zürich, Switzerland

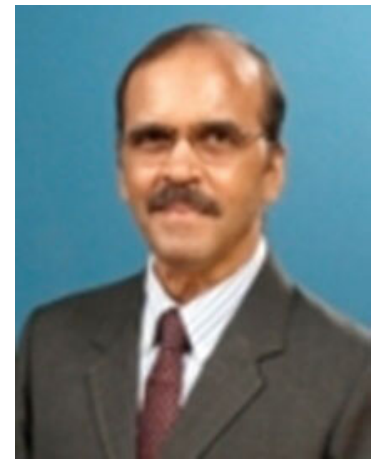

Krithi Ramamritham Department of Computer Science and Engineering, Indian Institute of Technology Bombay, Mumbai, India 


\section{Area Editors}

\section{Peer-to-Peer Data Management}

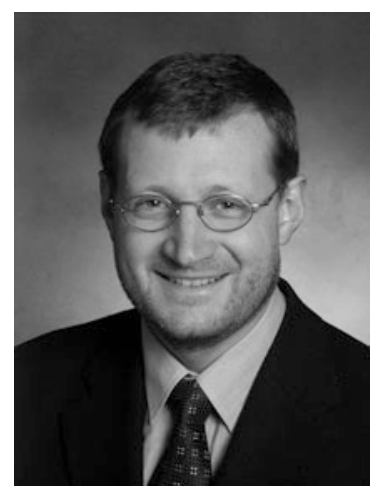

Karl Aberer Department of Computer Science, École Polytechnique Fédérale de Lausanne (EPFL), Lausanne, Switzerland

\section{Database Management System Architectures}

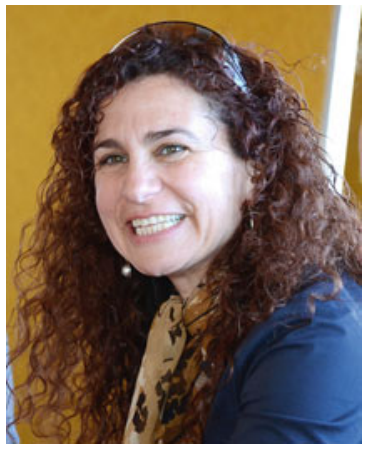

Anastasia Ailamaki Department of Computer Science, Ecole Polytechnique Fédérale de Lausanne, Lausanne, Switzerland 


\section{Information Retrieval Models}

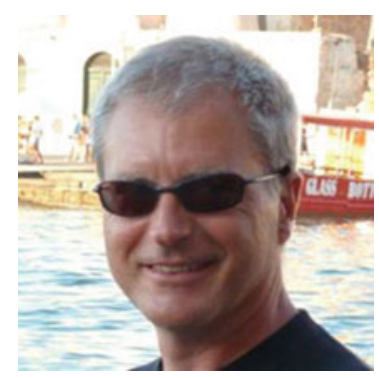

Giambattista Amati Fondazione Ugo Bordoni, Rome, Italy

\section{XML Data Management}

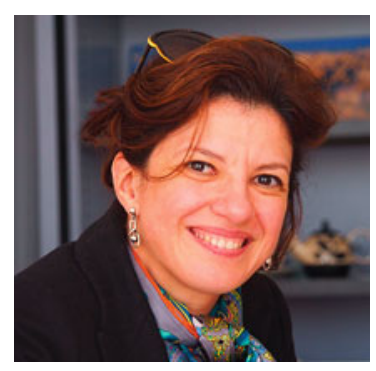

Sihem Amer-Yahia CNRS, University Grenoble Alpes, Saint Martin D'Hères, France

\section{Database Middleware}

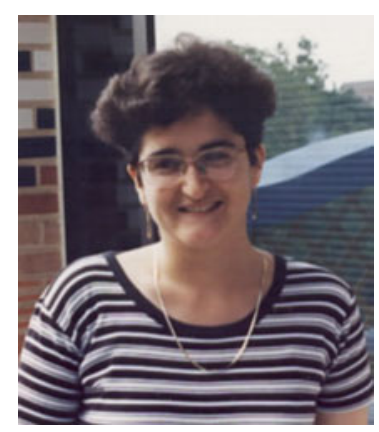

Cristiana Amza Electrical and Computer Engineering, University of Toronto, Toronto, ON, Canada 


\section{Database Management Utilities}

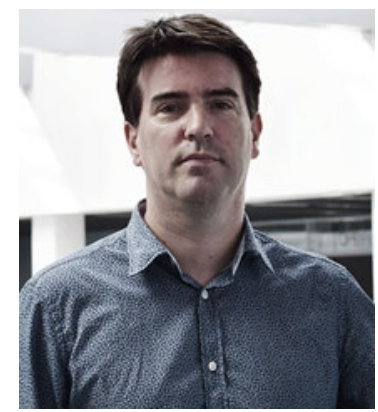

Philippe Bonnet Department of Computer Science, IT University of Copenhagen, Copenhagen, Denmark

\section{Visual Interfaces}

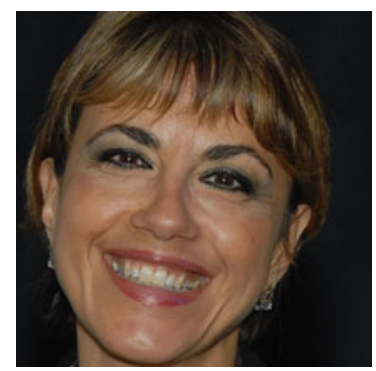

Tiziana Catarci Department of Computer Engineering, Automation and Management, Sapienza - Università di Roma, Rome, Italy

\section{Stream Data Management}

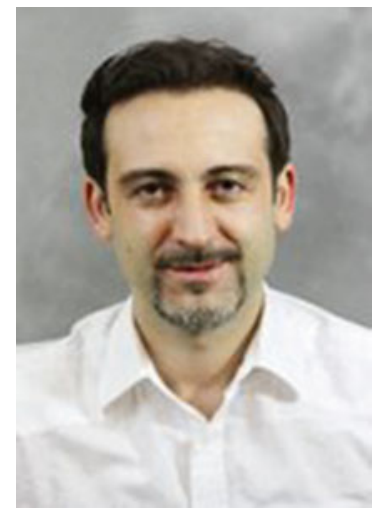

Ugur Cetintemel Department of Computer Science, Brown University, Providence, RI, USA 


\section{Querying over Data Integration Systems}

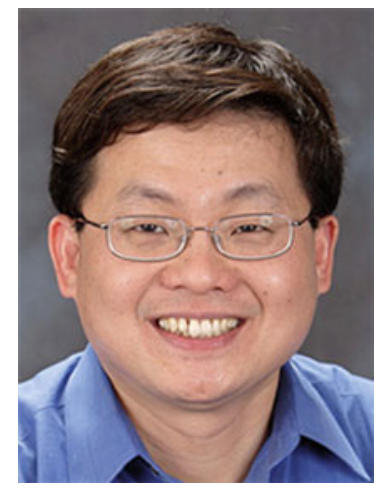

Kevin Chang Department of Computer Science, University of Illinois at Urbana-Champaign, Urbana-Champaign, IL, USA

\section{Self Management}

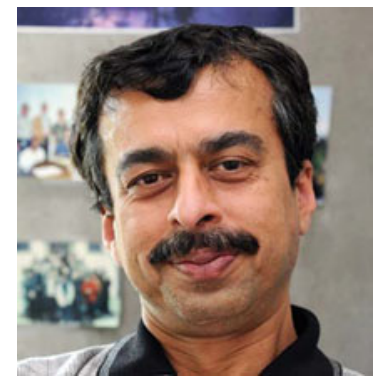

Surajit Chaudhuri Microsoft Corporation, Redmond, CA, USA

\section{Text Mining}

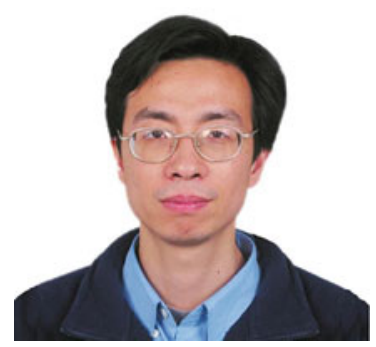

Zheng Chen Microsoft Corporation, Beijing, China 


\section{Extended Transaction Models}

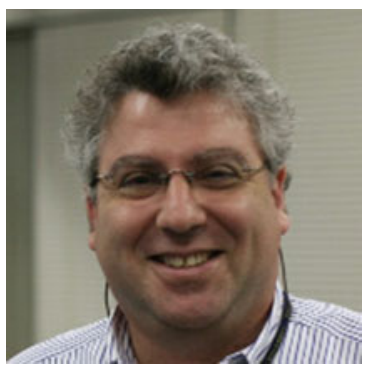

Panos K. Chrysanthis Department of Computer Science, School of Computing and Information, University of Pittsburgh, Pittsburgh, PA, USA

\section{Privacy-Preserving Data Mining}

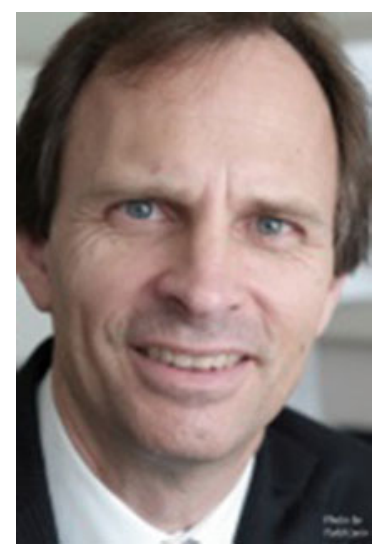

Chris Clifton Department of Computer Science, Purdue University, West Lafayette, IN, USA

\section{Digital Libraries}

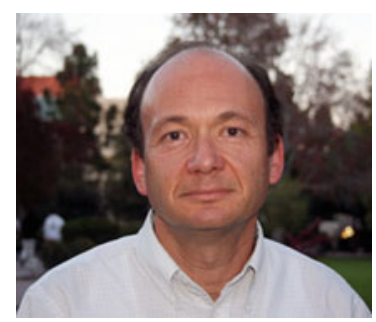

Amr El Abbadi Department of Computer Science, UC Santa Barbara, Santa Barbara, CA, USA 


\section{Data Models}

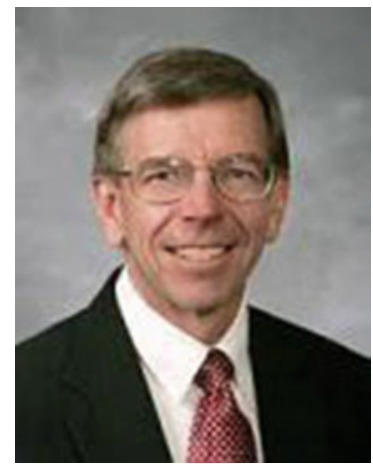

David W. Embley Department of Computer Science, Brigham Young University, Provo, UT, USA

\section{Complex Event Processing}

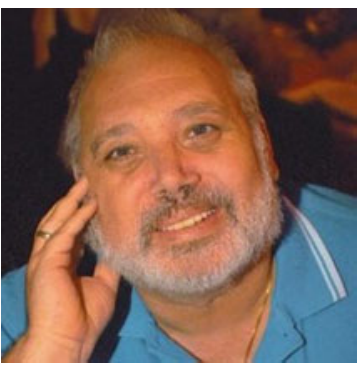

Opher Etzion Department of Information Systems, Yezreel Valley College, Jezreel Valley, Israel

\section{Database Security and Privacy}

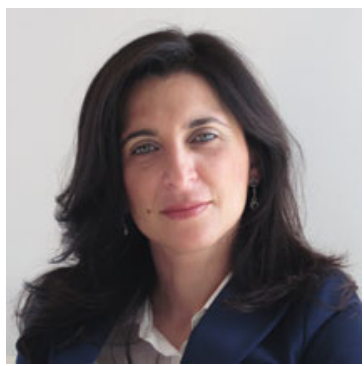

Elena Ferrari Department of Computer Science, Università degli Studi dell'Insubria, Varese, Italy 


\section{Semantic Web and Ontologies}

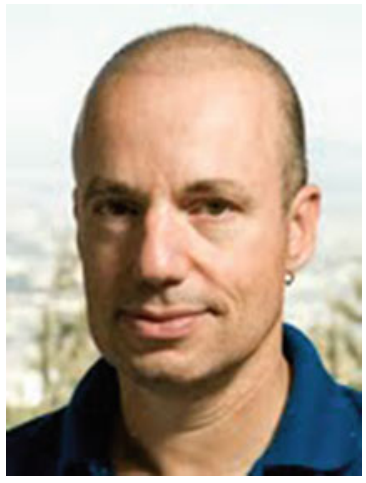

Avigdor Gal Industrial Engineering and Management, Technion - Israel Institute of Technology, Haifa, Israel

\section{Data Cleaning}

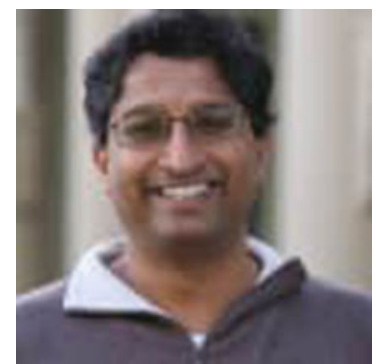

Venkatesh Ganti Alation, Redwood City, CA, USA

\section{Web Data Extraction}

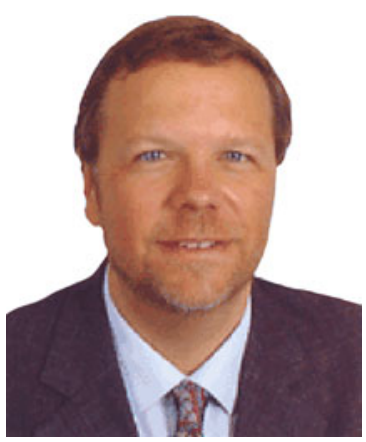

Georg Gottlob Computing Lab, Oxford University, Oxford, UK 


\section{Sensor Networks}

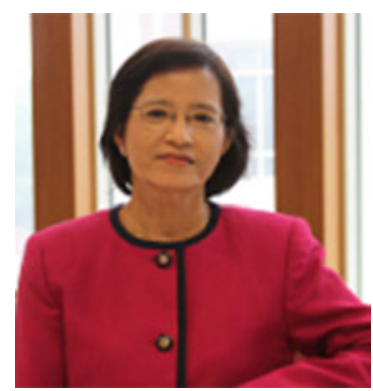

Le Gruenwald School of Computer Science, University of Oklahoma, Norman, OK, USA

\section{Data Clustering}

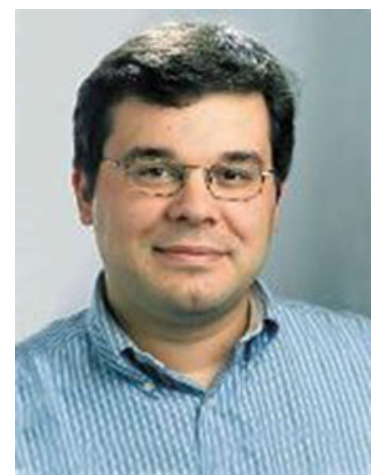

Dimitrios Gunopulos Department of Informatics and Telecommunications, National and Kapodistrian University of Athens, Athens, Greece

\section{Scientific Databases}

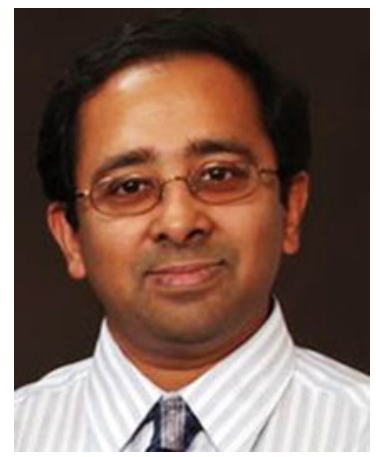

Amarnath Gupta San Diego Supercomputer Center, University of California San Diego, La Jolla, CA, USA 


\section{Geographical Information Systems}

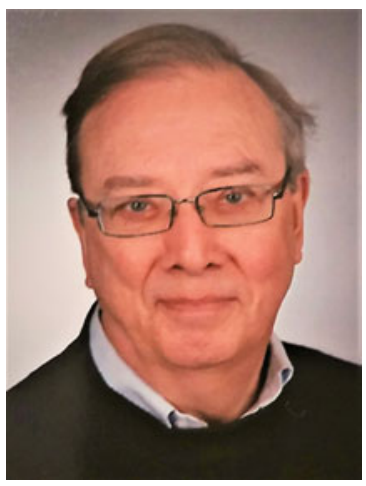

Ralf Hartmut Güting Department of Computer Science, FernUniversität in Hagen, Hagen, Germany

\section{Data Visualization}

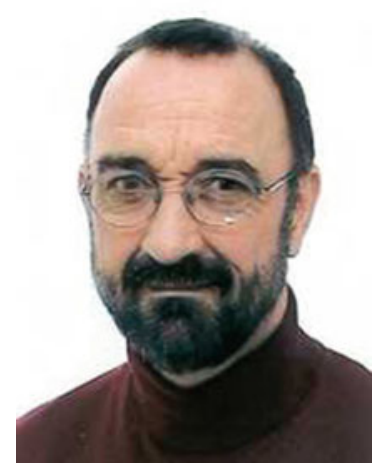

Hans Hinterberger Department of Computer Science, ETH Zurich, Zurich, Switzerland

\section{Web Services and Service Oriented Architectures}

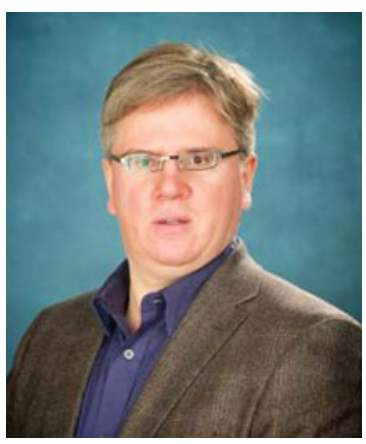

Hans-Arno Jacobsen Department of Electrical and Computer Engineering, University of Toronto, Toronto, ON, Canada 


\section{Metadata Management}

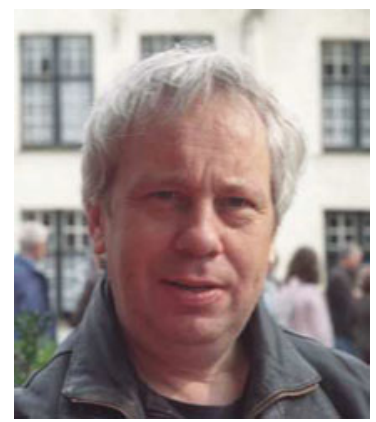

Manfred Jeusfeld IIT, University of Skövde, Skövde, Sweden

\section{Health Informatics Databases}

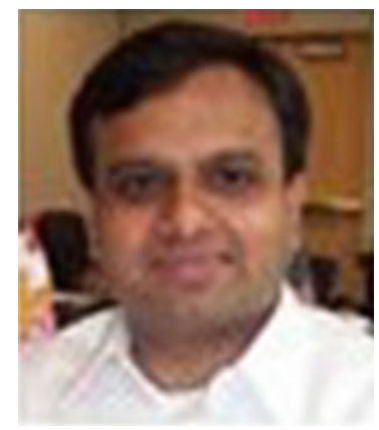

Vipul Kashyap CIGNA Healthcare, Bloomfield, CT, USA

\section{Visual Data Mining}

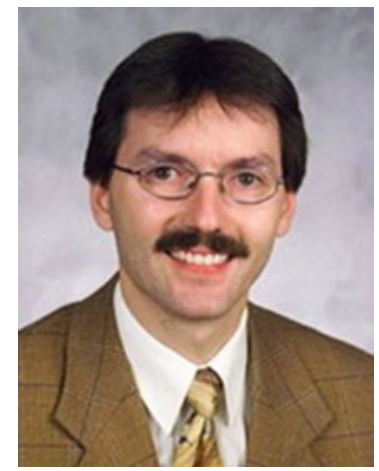

Daniel A. Keim Computer Science Department, University of Konstanz, Konstanz, Germany 


\section{Data Replication}

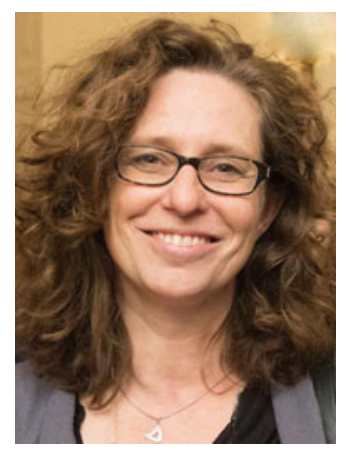

Bettina Kemme School of Computer Science, McGill University, Montreal, QC, Canada

\section{Storage Structures and Systems}

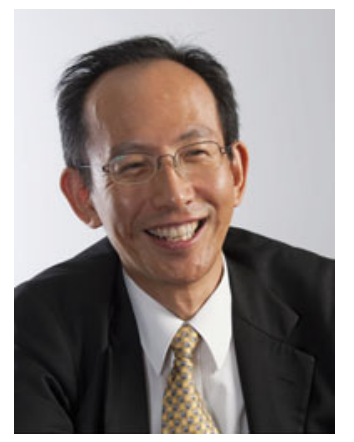

Masaru Kitsuregawa Institute of Industrial Science, University of Tokyo, Tokyo, Japan

\section{Views and View Management}

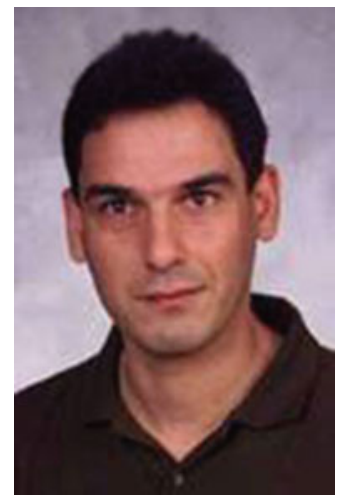

Yannis Kotidis Department of Informatics, Athens University of Economics and Business, Athens, Greece 


\section{Structured Text Retrieval}

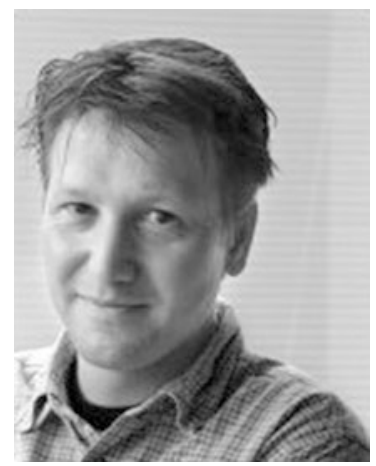

Jaap Kamps Faculty of Humanities, University of Amsterdam, Amsterdam, The Netherlands

\section{Information Quality}

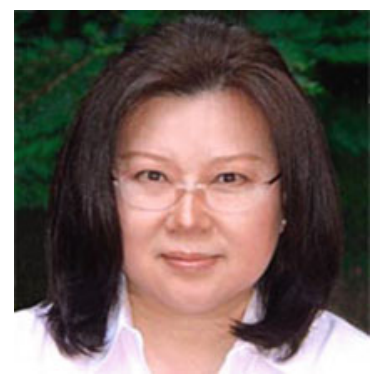

Yang W. Lee School of Business, Northeastern University, Boston, MA, USA

\section{Relational Theory}

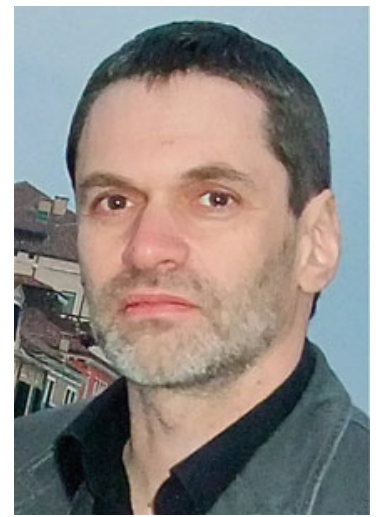

Leonid Libkin School of Informatics, University of Edinburgh, Edinburgh, UK 


\section{Information Retrieval Evaluation Measures}

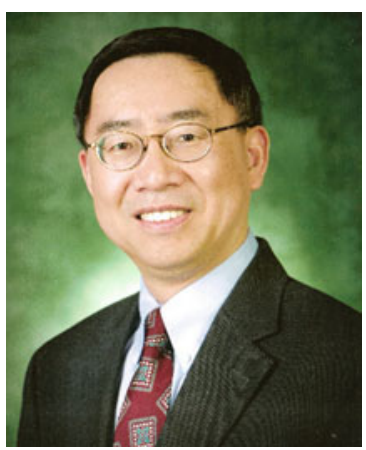

Weiyi Meng Department of Computer Science, State University of New York at Binghamton, Binghamton, NY, USA

\section{Logical Data Integration}

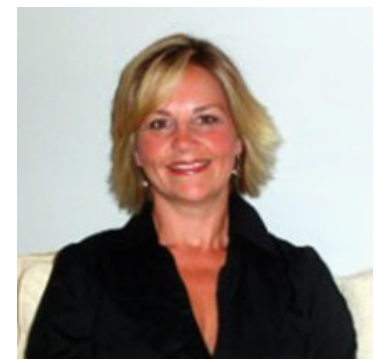

Renée J. Miller Department of Computer Science, University of Toronto, Toronto, ON, Canada

\section{Database Design}

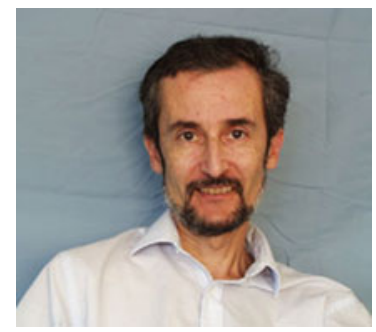

Alexander Borgida Department of Computer Science, Rutgers University, New Brunswick, NJ, USA 


\section{Text Indexing Techniques}

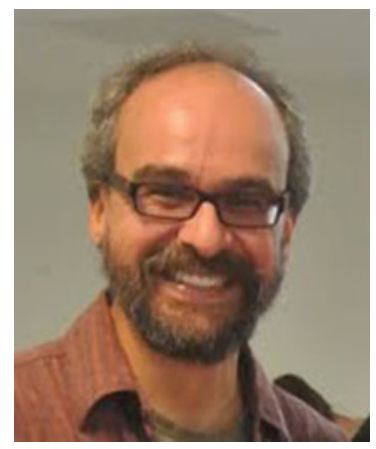

Mario A. Nascimento Department of Computing Science, University of Alberta, Edmonton, AB, Canada

\section{Data Quality}

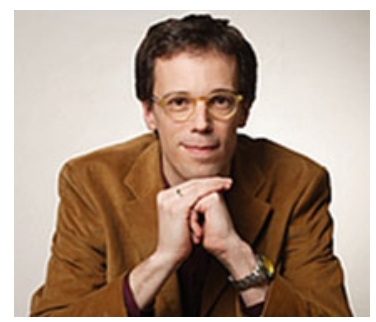

Felix Naumann Hasso Plattner Institute, University of Potsdam, Potsdam, Germany

\section{Web Search and Crawl}

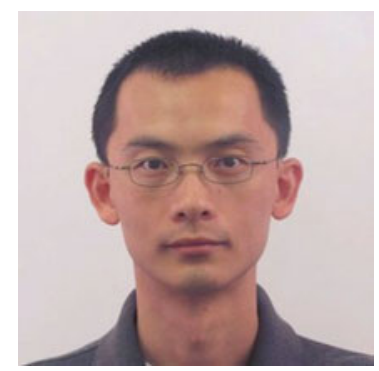

Cong Yu Google Research, New York, NY, USA 


\section{Multimedia Databases}

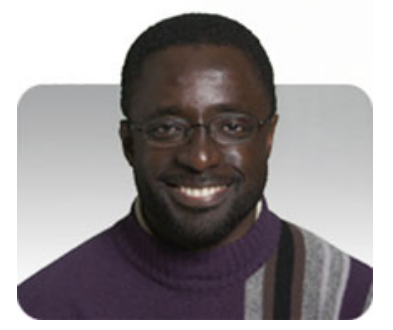

Vincent Oria Department of Computer Science, New Jersey Institute of Technology, Newark, NJ, USA

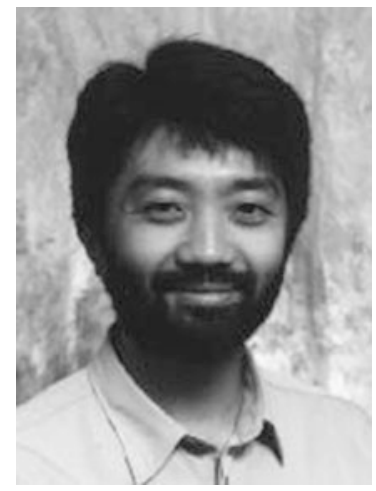

Shin'ichi Satoh Digital Content and Media Sciences ReseaMultimedia Information Research Division, National Institute of Informatics, Tokyo, Japan

\section{Active Databases}

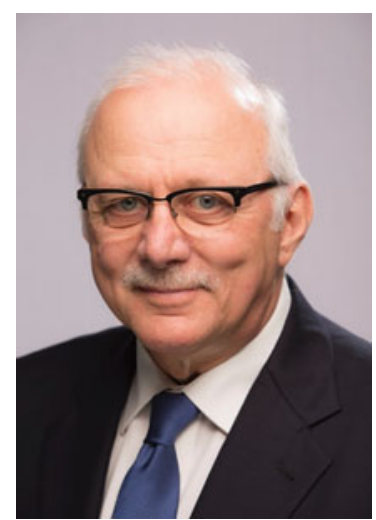

M. Tamer Özsu Cheriton School of Computer Science, University of Waterloo, Waterloo, ON, Canada 


\section{Spatial, Spatiotemporal, and Multidimensional Databases}

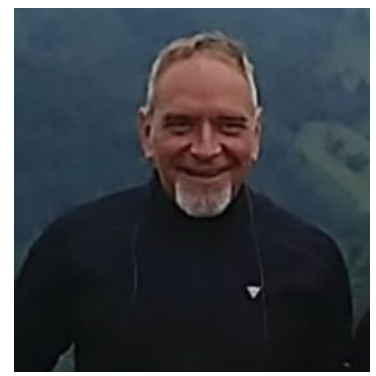

Dimitris Papadias Department of Computer Science and Engineering, Hong Kong University of Science and Technology, Kowloon, China

\section{Data Warehouse}

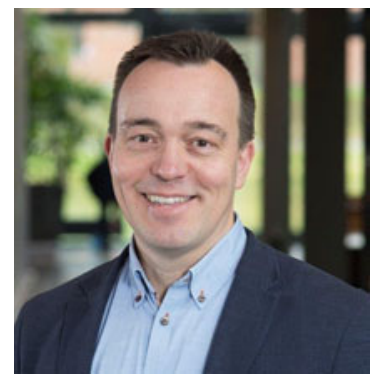

Torben Bach Pedersen Department of Computer Science, Aalborg University, Aalborg, Denmark

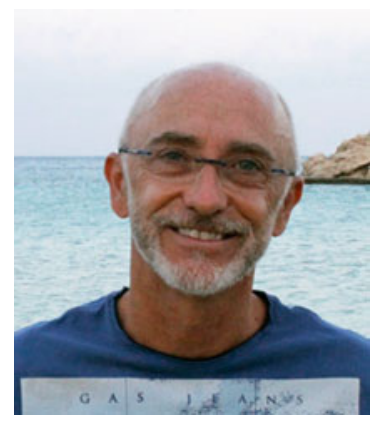

Stefano Rizzi DISI - University of Bologna, Bologna, Italy 


\section{Association Rule Mining}

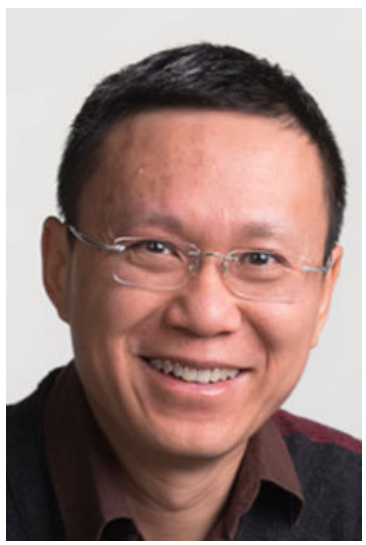

Jian Pei School of Computing Science, Simon Fraser University, Burnaby, BC, Canada

\section{Workflow Management}

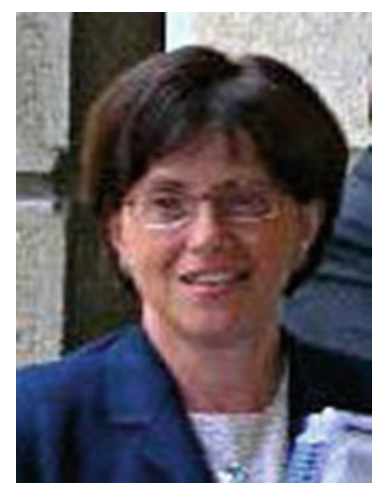

Barbara Pernici Department di Elettronica e Informazione, Politecnico di Milano, Milan, Italy

\section{Query Processing and Optimization}

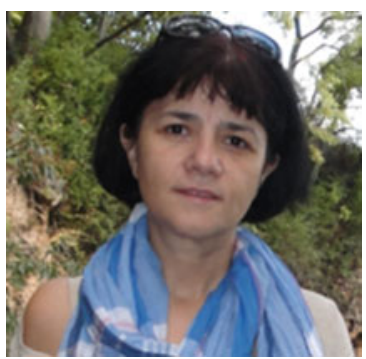

Evaggelia Pitoura Department of Computer Science and Engineering, University of Ioannina, Ioannina, Greece 


\section{Data Management for the Life Sciences}

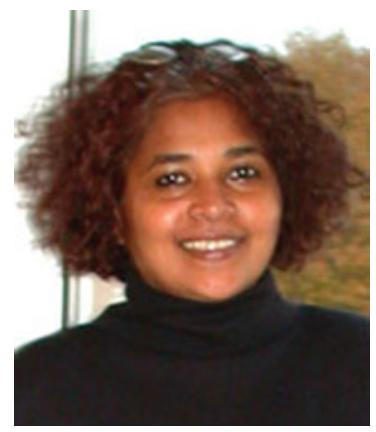

Louiqa Raschid Robert H. Smith School of Business, University of Maryland, College Park, MD, USA

\section{Information Retrieval Operations}

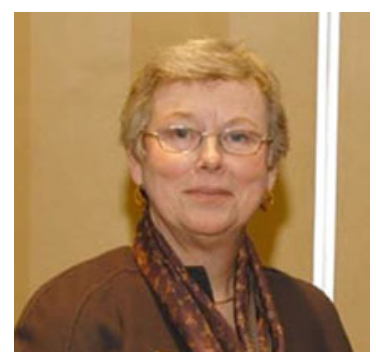

Edie Rasmussen Library, Archival and Information Studies, The University of British Columbia, VC, Canada

\section{Query Languages}

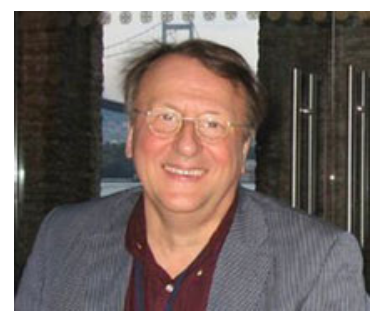

Tore Risch Department of Information Technology, Uppsala University, Uppsala, Sweden 


\section{Database Tuning and Performance}

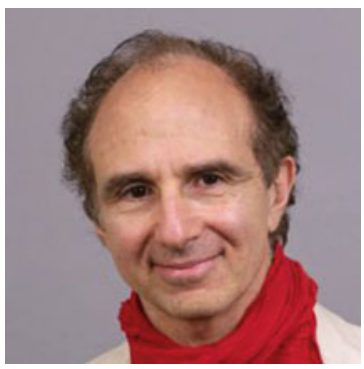

Dennis Shasha Department of Computer Science, New York University, New York, NY, USA

\section{Classification and Decision Trees}

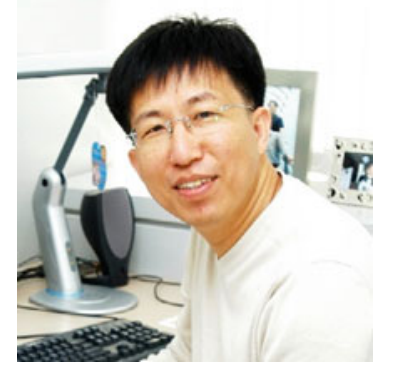

Kyuseok Shim School of Electrical Engineering and Computer Science, Seoul National University, Seoul, Republic of Korea

\section{Temporal Databases}

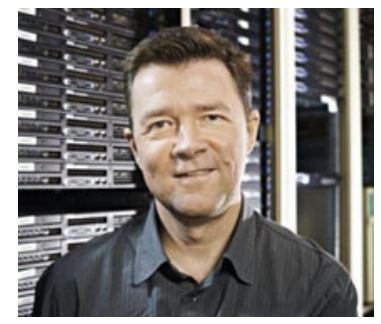

Christian S. Jensen Department of Computer Science, Aalborg University, Aalborg, Denmark 


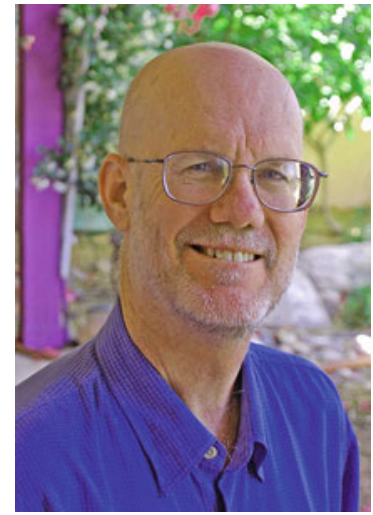

Richard T. Snodgrass Department of Computer Science, University of Arizona, Tucson, AZ, USA

\section{Stream Mining}

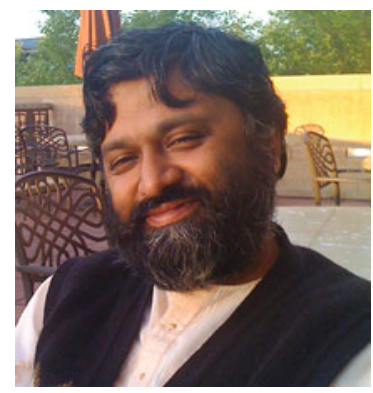

Divesh Srivastava AT\&T Labs-Research, Bedminster, NJ, USA

\section{Distributed Database Systems}

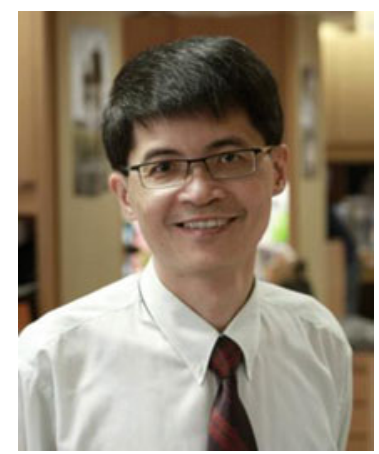

Kian-Lee Tan Department of Computer Science, National University of Singapore, Singapore, Singapore 


\section{Logics and Databases}

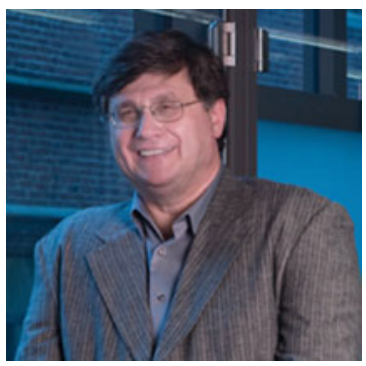

Val Tannen Department of Computer and Information Science, University of Pennsylvania, Philadelphia, PA, USA

\section{Structured and Semi-structured Document Databases}

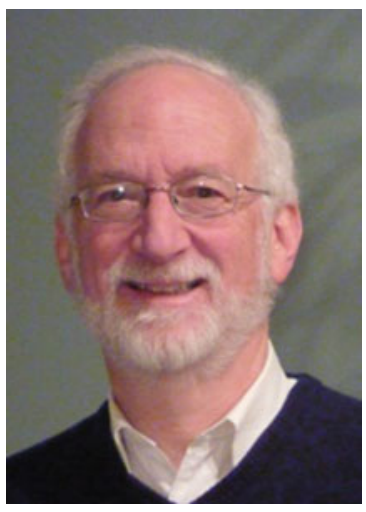

Frank Tompa David R. Cheriton School of Computer Science, University of Waterloo, Waterloo, ON, Canada

\section{Indexing}

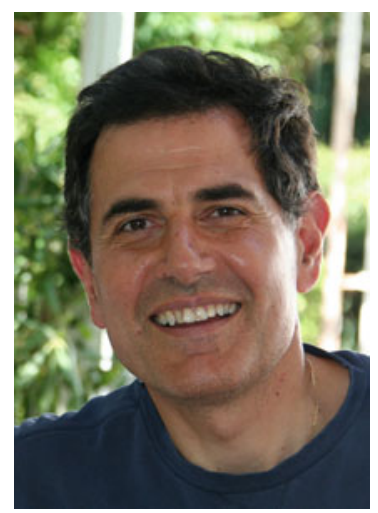

Vassilis J. Tsotras Department of Computer Science and Engineering, University of California-Riverside, Riverside, CA, USA 


\section{Parallel Database Systems}

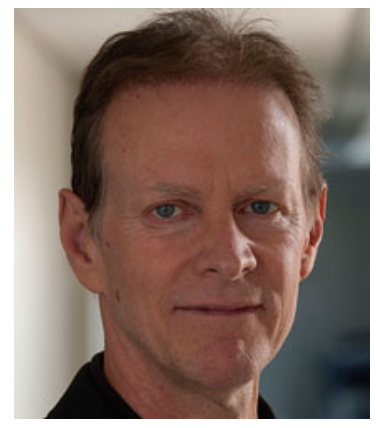

Patrick Valduriez INRIA and LIRMM, Montpellier, France

\section{Advanced Storage Systems}

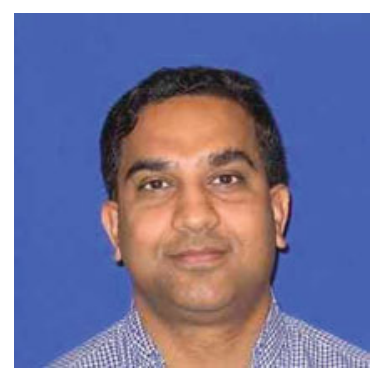

Kaladhar Voruganti Equinix, San Francisco, CA, USA

\section{Transaction Management}

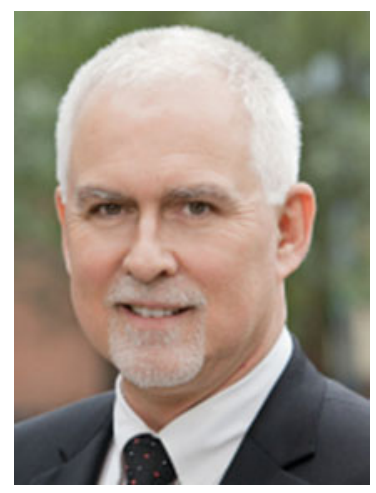

Gottfried Vossen Department of Information Systems, Westfälische Wilhelms-Universität, Münster, Germany 


\section{Mobile and Ubiquitous Data Management}

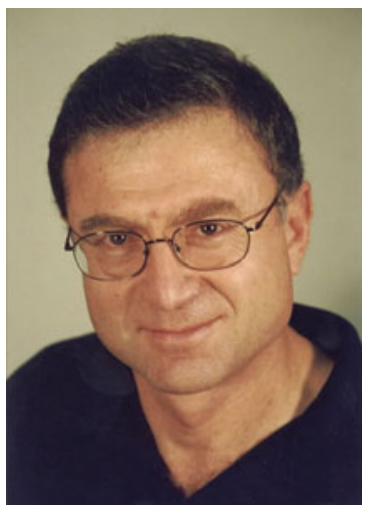

Ouri Wolfson Department of Computer Science, University of Illinois at Chicago, Chicago, IL, USA

\section{Multimedia Information Retrieval}

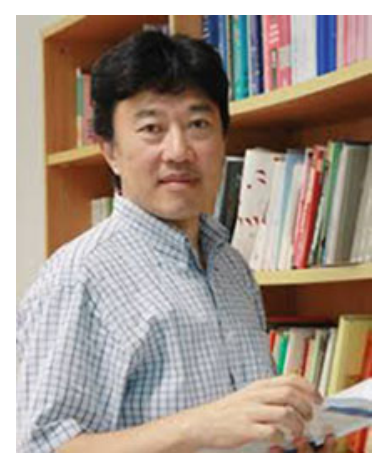

Jeffrey Xu Yu Department of Systems Engineering and Engineering Management, The Chinese University of Hong Kong, Hong Kong, China

\section{Approximation and Data Reduction Techniques}

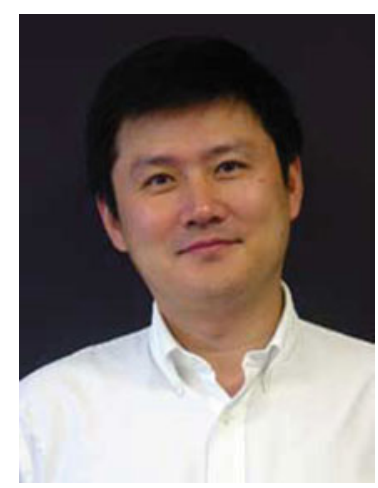

Xiaofang Zhou School of Information Technology and Electrical Engineering, University of Queensland, Brisbane, Australia 


\section{Social Networks}

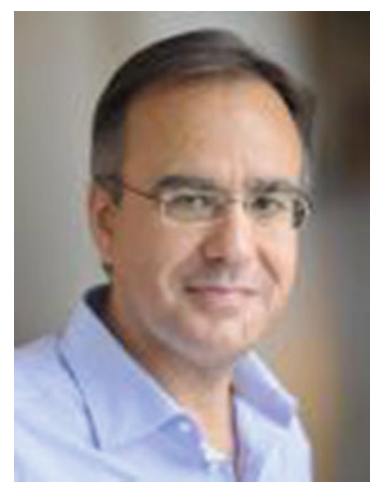

Nick Koudas Department of Computer Science, University of Toronto, Toronto, ON, Canada

\section{Cloud Data Management}

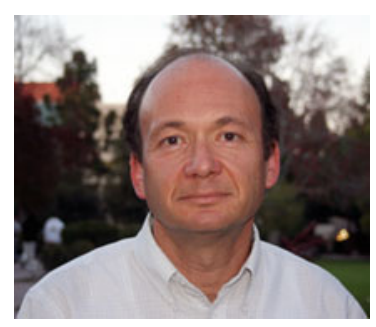

Amr El Abbadi Department of Computer Science, UC Santa Barbara, Santa Barbara, CA, USA

\section{Data Analytics}

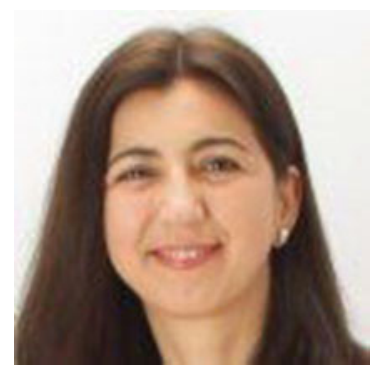

Fatma Özcan IBM Research - Almaden, San Jose, CA, USA 


\section{Data Management Fundamentals}

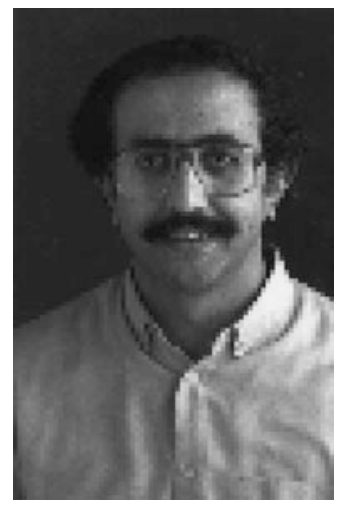

Ramez Elmasri Department of Computer Science and Engineering, The University of Texas at Arlington, Arlington, TX, USA

\section{NoSQL Databases}

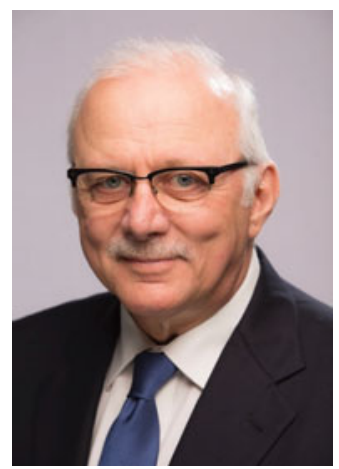

M. Tamer Özsu Cheriton School of Computer Science, University of Waterloo, Waterloo, ON, Canada

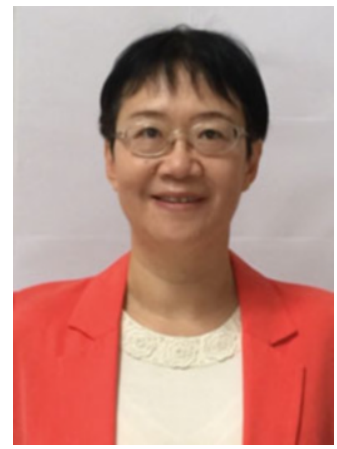

Ling Liu College of Computing, Georgia Institute of Technology, Atlanta, GA, USA 


\section{Graph Data Management}

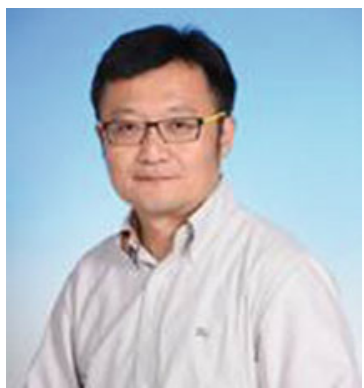

Lei Chen Department of Computer Science and Engineering, The Hong Kong University of Science and Technology, Hong Kong, China

\section{Data Provenance Management}

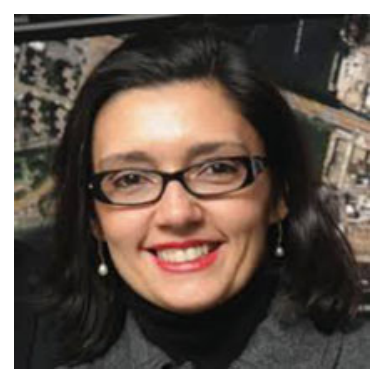

Juliana Freire Computer Science and Engineering, New York University, New York, NY, USA

\section{Ranking Queries}

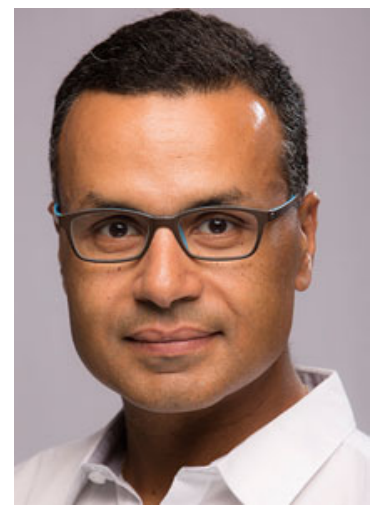

Ihab F. Ilyas Cheriton School of Computer Science, University of Waterloo, Waterloo, ON, Canada 


\section{Uncertain Data Management}

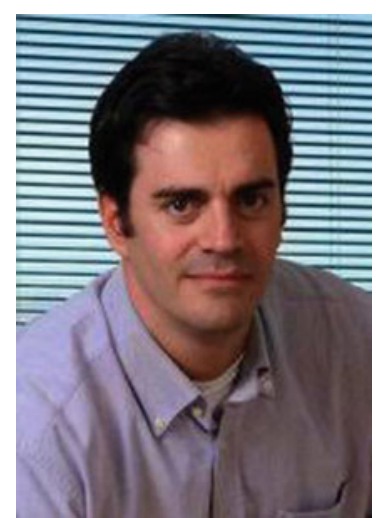

Minos Garofalakis Technical University of Crete, Chania, Greece

\section{Crowd Sourcing}

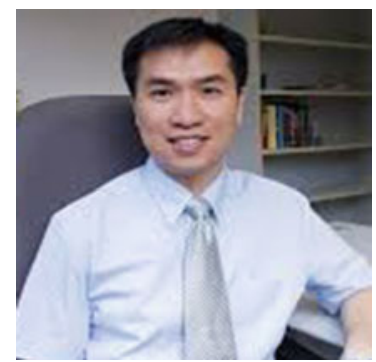

Reynold Cheng Computer Science, The University of Hong Kong, Hong Kong, China 


\section{List of Contributors}

Daniel Abadi Yale University, New Haven, CT, USA

Sofiane Abbar Qatar Computing Research Institute, Doha, Qatar

Alberto Abelló Polytechnic University of Catalonia, Barcelona, Spain

Serge Abiteboul Inria, Paris, France

Maribel Acosta Institute AIFB, Karlsruhe Institute of Technology, Karlsruhe, Germany

Ioannis Aekaterinidis University of Patras, Rio, Patras, Greece

Nitin Agarwal University of Arkansas, Little Rock, AR, USA

Charu C. Aggarwal IBM T. J. Watson Research Center, Yorktown Heights, NY, USA

Lalitha Agnihotri McGraw-Hill Education, New York, NY, USA

Marcos K. Aguilera VMware Research, Palo Alto, CA, USA

Yanif Ahmad Department of Computer Science, Brown University, Providence, RI, USA

Gail-Joon Ahn Arizona State University, Tempe, AZ, USA

Anastasia Ailamaki Informatique et Communications, Ecole Polytechnique Fédérale de Lausanne, Lausanne, Switzerland

Ablimit Aji Analytics Lab, Hewlett Packard, Palo Alto, CA, USA

Alexander Alexandrov Database and Information Management (DIMA), Institute of Software Engineering and Theoretical Computer Science, Berlin, Germany

Yousef J. Al-Houmaily Institute of Public Administration, Riyadh, Saudi Arabia

Mohammed Eunus Ali Department of Computer Science and Engineering, Bangladesh University of Engineering and Technology (BUET), Dhaka, Bangladesh

Robert B. Allen Drexel University, Philadelphia, PA, USA

Gustavo Alonso ETH Zürich, Zurich, Switzerland 
Omar Alonso Microsoft Silicon Valley, Mountain View, CA, USA

Bernd Amann Pierre \& Marie Curie University (UPMC), Paris, France

Giambattista Amati Fondazione Ugo Bordoni, Rome, Italy

Sihem Amer-Yahia CNRS, Univ. Grenoble Alps, Grenoble, France

Laboratoire d'Informatique de Grenoble, CNRS-LIG, Saint Martin-d'Hères, Grenoble, France

Rainer von Ammon Center for Information Technology Transfer $\mathrm{GmbH}$ (CITT), Regensburg, Germany

Robert A. Amsler CSC, Falls Church, VA, USA

Yael Amsterdamer Department of Computer Science, Bar Ilan University, Ramat Gan, Israel

Cristiana Amza Department of Electrical and Computer Engineering, University of Toronto, Toronto, ON, Canada

George Anadiotis VU University Amsterdam, Amsterdam, The Netherlands

Mihael Ankerst Ludwig-Maximilians-Universität München, Munich, Germany

Sameer Antani National Institutes of Health, Bethesda, MD, USA

Grigoris Antoniou Foundation for Research and Technology-Hellas (FORTH), Heraklion, Greece

Arvind Arasu Microsoft Research, Redmond, WA, USA

Danilo Ardagna Politechnico di Milano University, Milan, Italy

Walid G. Aref Purdue University, West Lafayette, IN, USA

Marcelo Arenas Pontifical Catholic University of Chile, Santiago, Chile

Nikos Armenatzoglou Department of Computer Science and Engineering, Hong Kong University of Science and Technology, Kowloon, Hong Kong, Hong Kong

Samuel Aronson Harvard Medical School - Partners Healthcare Center for Genetics and Genomics, Boston, MA, USA

Paavo Arvola University of Tampere, Tampere, Finland

Colin Atkinson Software Engineering, University of Mannheim, Mannheim, Germany

Noboru Babaguchi Osaka University, Osaka, Japan

Shivnath Babu Duke University, Durham, NC, USA

Nathan Backman Computer Science, Buena Vista University, Storm Lake, IA, USA 
Kenneth Paul Baclawski Northeastern University, Boston, MA, USA

Ricardo Baeza-Yates NTENT, USA - Univ. Pompeu Fabra, Spain - Univ. de Chile, Chile

James Bailey University of Melbourne, Melbourne, VIC, Australia

Peter Bailis Department of Computer Science, Stanford University, Palo Alto, CA, USA

Sumeet Bajaj Stony Brook University, Stony Brook, NY, USA

Peter Bak IBM Watson Health, Foundational Innovation, Haifa, Israel

Magdalena Balazinska University of Washington, Seattle, WA, USA

Krisztian Balog University of Stavanger, Stavanger, Norway

Farnoush Banaei-Kashani Computer Science and Engineering, University of Colorado Denver, Denver, CO, USA

Jie Bao Data Management, Analytics and Services (DMAS) and Ubiquitous Computing Group (Ubicomp), Microsoft Research Asia, Beijing, China

Stefano Baraldi University of Florence, Florence, Italy

Mauro Barbieri Phillips Research Europe, Eindhoven, The Netherlands

Denilson Barbosa University of Alberta, Edmonton, AL, Canada

Pablo Barceló University of Chile, Santiago, Chile

Luciano Baresi Dipartimento di Elettronica, Informazione e Bioingegneria - Politecnico di Milano, Milano, Italy

Ilaria Bartolini Department of Computer Science and Engineering (DISI), University of Bologna, Bologna, Italy

Saleh Basalamah Computer Science, Umm Al-Qura University, Mecca, Makkah Province, Saudi Arabia

Sugato Basu Google Inc, Mountain View, CA, USA

Carlo Batini University of Milano-Bicocca, Milan, Italy

Michal Batko Masaryk University, Brno, Czech Republic

Peter Baumann Jacobs University, Bremen, Germany

Robert Baumgartner Vienna University of Technology, Vienna, Austria

Sean Bechhofer University of Manchester, Manchester, UK

Steven M. Beitzel Telcordia Technologies, Piscataway, NJ, USA

Ladjel Bellatreche LIAS/ISAE-ENSMA, Poitiers University, Futuroscope, France

Omar Benjelloun Google Inc., New York, NY, USA

Véronique Benzaken University Paris 11, Orsay Cedex, France 
Rafael Berlanga Department of Computer Languages and Systems, Universitat Jaume I, Castellón, Spain

Mikael Berndtsson University of Skövde, The Informatics Research Centre, Skövde, Sweden

University of Skövde, School of Informatics, Skövde, Sweden

Philip A. Bernstein Microsoft Corporation, Redmond, WA, USA

Damon Andrew Berry University of Massachusetts, Lowell, MA, USA

Leopoldo Bertossi Carleton University, Ottawa, ON, Canada

Claudio Bettini Dipartimento di Informatica, Università degli Studi di Milano, Milan, Italy

Nigel Bevan Professional Usability Services, London, UK

Bharat Bhargava Purdue University, West Lafayette, IN, USA

Arnab Bhattacharya Indian Institute of Technology, Kanpur, India

Ernst Biersack Eurecom, Sophia Antipolis, France

Alberto Del Bimbo University of Florence, Florence, Italy

Carsten Binnig Computer Science-Database Systems, Brown University, Providence, RI, USA

Christian Bizer Web-based Systems Group, University of Mannheim, Mannheim, Germany

Alan F. Blackwell University of Cambridge, Cambridge, UK

Carlos Blanco GSyA and ISTR Research Groups, Department of Computer Science and Electronics, Faculty of Sciences, University of Cantabria, Santander, Spain

Marina Blanton University of Notre Dame, Notre Dame, IN, USA

Toine Bogers Department of Communication and Psychology, Aalborg University Copenhagen, Copenhagen, Denmark

Philip Bohannon Yahoo! Research, Santa Clara, CA, USA

Michael H. Böhlen Free University of Bozen-Bolzano, Bozen-Bolzano, Italy

University of Zurich, Zürich, Switzerland

Christian Böhm University of Munich, Munich, Germany

Peter Boncz CWI, Amsterdam, The Netherlands

Philippe Bonnet Department of Computer Science, IT University of Copenhagen, Copenhagen, Denmark

Alexander Borgida Rutgers University, New Brunswick, NJ, USA 
Vineyak Borkar CTO and VP of Engineering, X15 Software, San Francisco, CA, USA

Chavdar Botev Yahoo Research!, Cornell University, Ithaca, NY, USA

Sara Bouchenak University of Grenoble I - INRIA, Grenoble, France

Luc Bouganim INRIA Saclay and UVSQ, Le Chesnay, France

Nozha Boujemaa INRIA Paris-Rocquencourt, Le Chesnay, France

Shawn Bowers University of California-Davis, Davis, CA, USA

Stéphane Bressan National University of Singapore, School of Computing, Department of Computer Science, Singapore, Singapore

Martin Breunig University of Osnabrueck, Osnabrueck, Germany

Scott A. Bridwell University of Utah, Salt Lake City, UT, USA

Thomas Brinkhoff Institute for Applied Photogrammetry and Geoinformatics (IAPG), Oldenburg, Germany

Nieves R. Brisaboa Database Laboratory, Department of Computer Science, University of A Coruña, A Coruña, Spain

Andrei Broder Yahoo! Research, Santa Clara, CA, USA

Nicolas Bruno Microsoft Corporation, Redmond, WA, USA

François Bry University of Munich, Munich, Germany

Yingyi Bu Chinese University of Hong Kong, Hong Kong, China

Alejandro Buchmann Darmstadt University of Technology, Darmstadt, Germany

Thilina Buddhika Colorado State University, Fort Collins, CO, USA

Chiranjeeb Buragohain Amazon.com, Seattle, WA, USA

Thorsten Büring Ludwig-Maximilians-University Munich, Munich, Germany

Benjamin Bustos Department of Computer Science, University of Chile, Santiago, Chile

David J. Buttler Lawrence Livermore National Laboratory, Livermore, CA, USA

Yanli Cai Shanghai Jiao Tong University, Shanghai, China

Diego Calvanese Research Centre for Knowledge and Data (KRDB), Free University of Bozen-Bolzano, Bolzano, Italy

Guadalupe Canahuate The Ohio State University, Columbus, OH, USA

K. Selcuk Candan Arizona State University, Tempe, AZ, USA

Turkmen Canli University of Illinois at Chicago, Chicago, IL, USA 
Alan Cannon Napier University, Edinburgh, UK

Cornelia Caragea Computer Science and Engineering, University of North Texas, Denton, TX, USA

Barbara Carminati Department of Theoretical and Applied Science, University of Insubria, Varese, Italy

Sheelagh Carpendale University of Calgary, Calgary, AB, Canada

Michael W. Carroll Villanova University School of Law, Villanova, PA, USA

Ben Carterette University of Massachusetts Amherst, Amherst, MA, USA

Marco A. Casanova Pontifical Catholic University of Rio de Janeiro, Rio de Janeiro, Brazil

Giuseppe Castagna C.N.R.S. and University Paris 7, Paris, France

Tiziana Catarci Dipartimento di Ingegneria Informatica, Automatica e Gestionale “A.Ruberti”, Sapienza - Università di Roma, Rome, Italy

James Caverlee Department of Computer Science, Texas A\&M University, College Station, TX, USA

Emmanuel Cecchet EPFL, Lausanne, Switzerland

Wojciech Cellary Department of Information Technology, Poznan University of Economics, Poznan, Poland

Ana Cerdeira-Pena Database Laboratory, Department of Computer Science, University of A Coruña, A Coruña, Spain

Michal Ceresna Lixto Software GmbH, Vienna, Austria

Ugur Cetintemel Department of Computer Science, Brown University, Providence, RI, USA

Soumen Chakrabarti Indian Institute of Technology of Bombay, Mumbai, India

Don Chamberlin IBM Almaden Research Center, San Jose, CA, USA

Allen Chan IBM Toronto Software Lab, Markham, ON, Canada

Chee-Yong Chan National University of Singapore, Singapore, Singapore

K. Mani Chandy California Institute of Technology, Pasadena, CA, USA

Edward Y. Chang Google Research, Mountain View, CA, USA

Kevin Chang Department of Computer Science, University of Illinois at Urbana-Champaign, Urbana, IL, USA

Adriane Chapman University of Southampton, Southampton, UK

Surajit Chaudhuri Microsoft Research, Microsoft Corporation, Redmond, WA, USA 
Elizabeth S. Chen Partners HealthCare System, Boston, MA, USA

James L. Chen University of Chicago, Chicago, IL, USA

Jin Chen Computer Engineering Research Group, University of Toronto, Toronto, ON, Canada

Jinjun Chen Swinburne University of Technology, Melbourne, VIC, Australia

Jinchuan Chen Key Laboratory of Data Engineering and Knowledge Engineering, Ministry of Education, Renmin University of China, Beijing

Lei Chen Hong Kong University of Science and Technology, Hong Kong, China

Peter P. Chen Louisiana State University, Baton Rouge, LA, USA

James Cheney University of Edinburgh, Edinburgh, UK

Hong Cheng Department of Systems Engineering and Engineering Management, The Chinese University of Hong Kong, Hong Kong, China

Reynold Cheng Computer Science, The University of Hong Kong, Hong Kong, China

Vivying S. Y. Cheng Hong Kong University of Science and Technology, Hong Kong, China

InduShobha N. Chengalur-Smith University at Albany - SUNY, Albany, NY, USA

Mitch Cherniack Brandeis University, Wattham, MA, USA

Yun Chi NEC Laboratories America, Cupertino, CA, USA

Fernando Chirigati NYU Tandon School of Engineering, Brooklyn, NY, USA

Rada Chirkova North Carolina State University, Raleigh, NC, USA

Laura Chiticariu Scalable Natural Language Processing, IBM Research Almaden, San Jose, CA, USA

Jan Chomicki Department of Computer Science and Engineering, State University of New York at Buffalo, Buffalo, NY, USA

Fred Chong Computer Science, University of Chicago, Chicago, IL, USA

Stephanie Chow University of Ontario Institute of Technology, Oshawa, ON, Canada

Peter Christen Research School of Computer Science, The Australian National University, Canberra, Australia

Vassilis Christophides INRIA Paris-Roquencourt, Paris, France 
Panos K. Chrysanthis Department of Computer Science, University of Pittsburgh, Pittsburgh, PA, USA

Paolo Ciaccia Computer Science and Engineering, University of Bologna, Bologna, Italy

John Cieslewicz Google Inc., Mountain View, CA, USA

Gianluigi Ciocca University of Milano-Bicocca, Milan, Italy

Eugene Clark Harvard Medical School - Partners Healthcare Center for Genetics and Genomics, Boston, MA, USA

Charles L. A. Clarke University of Waterloo, Waterloo, ON, Canada

William R. Claycomb CERT Insider Threat Center, Software Engineering Institute, Carnegie Mellon University, Pittsburgh, PA, USA

Eliseo Clementini University of L'Aguila, L'Aguila, Italy

Chris Clifton Department of Computer Science, Purdue University, West Lafayette, IN, USA

Edith Cohen AT\&T Labs-Research, Florham Park, NJ, USA

Sara Cohen The Rachel and Selim Benin School of Computer Science and Engineering, The Hebrew University of Jerusalem, Jerusalem, Israel

Sarah Cohen-Boulakia University Paris-Sud, Orsay Cedex, France

Carlo Combi Department of Computer Science, University of Verona, Verona, VR, Italy

Mariano P. Consens University of Toronto, Toronto, ON, Canada

Dianne Cook Iowa State University, Ames, IA, USA

Graham Cormode Computer Science, University of Warwick, Warwick, UK

Antonio Corral University of Almeria, Almeria, Spain

Maria Francesca Costabile Department of Computer Science, University of Bari, Bari, Italy

Nick Craswell Microsoft Research Cambridge, Cambridge, UK

Fabio Crestani University of Lugano, Lugano, Switzerland

Marco Antonio Cristo FUCAPI, Manaus, Brazil

Maxime Crochemore King's College London, London, UK

Université Paris-Est, Paris, France

Andrew Crotty Database Group, Brown University, Providence, RI, USA

Matthew G. Crowson University of Chicago, Chicago, IL, USA

Michel Crucianu Conservatoire National des Arts et Métiers, Paris, France 
Philippe Cudré-Mauroux Massachusetts Institute of Technology, Cambridge, MA, USA

Sonia Leila Da Silva Cerveteri, Italy

Peter Dadam University of Ulm, Ulm, Germany

Mehmet M. Dalkiliç Indiana University, Bloomington, IN, USA

Nilesh Dalvi Airbnb, San Francisco, CA, USA

Marina Danilevsky IBM Almaden Research Center, San Jose, CA, USA

Minh Dao-Tran Institute of Information Systems, Vienna University of Technology, Vienna, Austria

Gautam Das Department of Computer Science and Engineering, University of Texas at Arlington, Arlington, TX, USA

Mahashweta Das Visa Research, Palo Alto, CA, USA

Sudipto Das Microsoft Research, Redmond, WA, USA

Manoranjan Dash Nanyang Technological University, Singapore, Singapore

Anupam Datta Computer Science Department and Electrical and Computer Engineering Department, Carnegie Mellon University, Pittsburgh, PA, USA

Anwitaman Datta Nanyang Technological University, Singapore, Singapore

Ian Davidson University of California-Davis, Davis, CA, USA

Susan B. Davidson Department of Computer and Information Science, University of Pennsylvania, Philadelphia, PA, USA

Todd Davis Department of Computer Science and Software Engineering, Concordia University, Montreal, QC, Canada

Maria De Marsico Sapienza University of Rome, Rome, Italy

Edleno Silva De Moura Federal University of Amazonas, Manaus, Brazil

Antonios Deligiannakis University of Athens, Athens, Greece

Alex Delis University of Athens, Athens, Greece

Alan Demers Cornell University, Ithaca, NY, USA

Jennifer Dempsey University of Arizona, Tucson, AZ, USA

Raytheon Missile Systems, Tucson, AZ, USA

Ke Deng University of Queensland, Brisbane, QLD, Australia

Amol Deshpande University of Maryland, College Park, MD, USA

Zoran Despotovic NTT DoCoMo Communications Laboratories Europe, Munich, Germany 
Alin Deutsch University of California-San Diego, La Jolla, CA, USA

Yanlei Diao University of Massachusetts Amherst, Amherst, MA, USA

Suzanne W. Dietrich Arizona State University, Phoenix, AZ, USA

Nevenka Dimitrova Philips Research, Briarcliff Manor, New York, USA

Bolin Ding University of Illinois at Urbana-Champaign, Urbana, IL, USA

Chris Ding University of Texas at Arlington, Arlington, TX, USA

Alan Dix Lancaster University, Lancaster, UK

Belayadi Djahida National High School for Computer Science (ESI), Algiers, Algeria

Hong-Hai Do SAP AG, Dresden, Germany

Gillian Dobbie University of Auckland, Auckland, New Zealand

Alin Dobra University of Florida, Gainesville, FL, USA

Vlastislav Dohnal Masaryk University, Brno, Czech Republic

Mario Döller University of Applied Science Kufstein, Kufstein, Austria

Carlotta Domeniconi George Mason University, Fairfax, VA, USA

Josep Domingo-Ferrer Universitat Rovira i Virgili, Tarragona, Catalonia, Spain

Guozhu Dong Wright State University, Dayton, OH, USA

Xin Luna Dong Amazon, Seattle, WA, USA

Chitra Dorai IBM T. J. Watson Research Center, Hawthorne, NY, USA

Zhicheng Dou Nankai University, Tianjin, China

Ahlame Douzal CNRS, Univ. Grenoble Alps, Grenoble, France

Yang Du Northeastern University, Boston, MA, USA

Susan Dumais Microsoft Research, Redmond, WA, USA

Marlon Dumas University of Tartu, Tartu, Estonia

Schahram Dustdar Technical University of Vienna, Vienna, Austria

Curtis E. Dyreson Utah State University, Logan, UT, USA

Johann Eder Department of Informatics-Systems, Alpen-Adria-Universität Klagenfurt, Klagenfurt, Austria

Milad Eftekhar University of Toronto, Toronto, ON, Canada

Thomas Eiter Institute of Information Systems, Vienna University of Technology, Vienna, Austria

Ibrahim Abu El-Khair Information Science Department, School of Social Sciences, Umm Al-Qura University, Mecca, Saudi Arabia 
Ahmed K. Elmagarmid Purdue University, West Lafayette, IN, USA

Qatar Computing Research Institute, HBKU, Doha, Qatar

Ramez Elmasri Computer Science, The University of Texas at Arlington, Arlington, TX, USA

Aaron J. Elmore Department of Computer Science, University of Chicago, Chicago, IL, USA

Sameh Elnikety Microsoft Research, Redmond, WA, USA

David W. Embley Brigham Young University, Provo, UT, USA

Vincent Englebert University of Namur, Namur, Belgium

AnnMarie Ericsson University of Skövde, Skövde, Sweden

Martin Ester Simon Fraser University, Burnaby, BC, Canada

Opher Etzion IBM Software Group, IBM Haifa Labs, Haifa University Campus, Haifa, Israel

Patrick Eugster Purdue University, West Lafayette, IN, USA

Ronald Fagin IBM Almaden Research Center, San Jose, CA, USA

Ju Fan DEKE Lab and School of Information, Renmin University of China, Beijing, China

Wei Fan IBM T.J. Watson Research, Hawthorne, NY, USA

Wenfei Fan University of Edinburgh, Edinburgh, UK

Beihang University, Beijing, China

Hui Fang University of Delaware, Newark, DE, USA

Alan Fekete University of Sydney, Sydney, NSW, Australia

Jean-Daniel Fekete INRIA, LRI University Paris Sud, Orsay Cedex, France

Pascal Felber University of Neuchatel, Neuchatel, Switzerland

Paolino Di Felice University of L'Aguila, L'Aguila, Italy

Hakan Ferhatosmanoglu The Ohio State University, Columbus, OH, USA

Eduardo B. Fernandez Florida Atlantic University, Boca Raton, FL, USA

Eduardo Fernández-Medina GSyA Research Group, Department of Information Technologies and Systems, Institute of Information Technologies and Systems, Escuela Superior de Informática, University of Castilla-La Mancha, Ciudad Real, Spain

Paolo Ferragina Department of Computer Science, University of Pisa, Pisa, Italy

Elena Ferrari DiSTA, University of Insubria, Varese, Italy

Dennis Fetterly Google, Inc., Mountain View, CA, USA 
Stephen E. Fienberg Carnegie Mellon University, Pittsburgh, PA, USA

Michael Fink Institute of Information Systems, Vienna University of Technology, Vienna, Austria

Peter M. Fischer Computer Science Department, University of Freiburg, Freiburg, Germany

Simone Fischer-Hübner Karlstad University, Karlstad, Sweden

Fabian Flöck GESIS - Leibniz Institute for the Social Sciences, Köln, Germany

Avrilia Floratou Microsoft, Sunnyvale, CA, USA

Leila De Floriani University of Genova, Genoa, Italy

Christian Fluhr CEA LIST, Fontenay-aux, Roses, France

Greg Flurry IBM SOA Advanced Technology, Armonk, NY, USA

Edward A. Fox Virginia Tech, Blacksburg, VA, USA

Chiara Francalanci Politecnico di Milano University, Milan, Italy

Andrew U. Frank Vienna University of Technology, Vienna, Austria

Michael J. Franklin University of California-Berkeley, Berkeley, CA, USA

Keir Fraser University of Cambridge, Cambridge, UK

Juliana Freire NYU Tandon School of Engineering, Brooklyn, NY, USA

NYU Center for Data Science, New York, NY, USA

New York University, New York, NY, USA

Elias Frentzos University of Piraeus, Piraeus, Greece

Johann-Christoph Freytag Humboldt University of Berlin, Berlin, Germany

Ophir Frieder Georgetown University, Washington, DC, USA

Oliver Frölich Lixto Software GmbH, Vienna, Austria

Ada Wai-Chee Fu Chinese University of Hong Kong, Hong Kong, China

Xiang Fu University of Southern California, Los Angeles, CA, USA

Kazuhisa Fujimoto Hitachi Ltd., Tokyo, Japan

Tim Furche University of Munich, Munich, Germany

Ariel Fuxman Microsoft Research, Mountain View, CA, USA

Silvia Gabrielli Bruno Kessler Foundation, Trento, Italy

Isabella Gagliardi National Research Council (CNR), Milan, Italy

Avigdor Gal Faculty of Industrial Engineering and Management, TechnionIsrael Institute of Technology, Haifa, Israel 
Alex Galakatos Database Group, Brown University, Providence, RI, USA

Department of Computer Science, Brown University, Providence, RI, USA

Wojciech Galuba EPFL, Lausanne, Switzerland

Johann Gamper Free University of Bozen-Bolzano, Bolzano, Italy

Weihao Gan University of Southern California, Los Angeles, CA, USA

Vijay Gandhi University of Minnesota, Minneapolis, MN, USA

Venkatesh Ganti Microsoft Research, Microsoft Corporation, Redmond, WA, USA

Dengfeng Gao IBM Silicon Valley Lab, San Jose, CA, USA

Like Gao Teradata Corporation, San Diego, CA, USA

Wei Gao Qatar Computing Research Institute, Doha, Qatar

Minos Garofalakis Technical University of Crete, Chania, Greece

Wolfgang Gatterbauer University of Washington, Seattle, WA, USA

Buğra Gedik Department of Computer Engineering, Bilkent University, Ankara, Turkey

IBM T.J. Watson Research Center, Hawthorne, NY, USA

Floris Geerts University of Antwerp, Antwerp, Belgium

Johannes Gehrke Cornell University, Ithaca, NY, USA

Betsy George Oracle (America), Nashua, NH, USA

Lawrence Gerstley PSMI Consulting, San Francisco, CA, USA

Michael Gertz Heidelberg University, Heidelberg, Germany

Giorgio Ghelli Dipartimento di Informatica, Università di Pisa, Pisa, Italy

Gabriel Ghinita National University of Singapore, Singapore, Singapore

Giuseppe De Giacomo Dip. di Ingegneria Informatica Automatica e Gestionale Antonio Ruberti, Sapienza Università di Roma, Rome, Italy

Phillip B. Gibbons Computer Science Department and the Electrical and Computer Engineering Department, Carnegie Mellon University, Pittsburgh, PA, USA

Sarunas Girdzijauskas EPFL, Lausanne, Switzerland

Fausto Giunchiglia University of Trento, Trento, Italy

Kazuo Goda The University of Tokyo, Tokyo, Japan

Max Goebel Vienna University of Technology, Vienna, Austria

Bart Goethals University of Antwerp, Antwerp, Belgium

Martin Gogolla University of Bremen, Bremen, Germany 
Aniruddha Gokhale Vanderbilt University, Nashville, TN, USA

Lukasz Golab University of Waterloo, Waterloo, ON, Canada

Matteo Golfarelli DISI - University of Bologna, Bologna, Italy

Arturo González-Ferrer Innovation Unit, Instituto de Investigación Sanitaria del Hospital Clínico San Carlos (IdISSC), Madrid, Spain

Michael F. Goodchild University of California-Santa Barbara, Santa Barbara, CA, USA

Georg Gottlob Computing Laboratory, Oxford University, Oxford, UK

Valerie Gouet-Brunet CNAM Paris, Paris, France

Ramesh Govindan University of Southern California, Los Angeles, CA, USA

Tyrone Gradison Proficiency Labs, Ashland, OR, USA

Goetz Graefe Google, Inc., Mountain View, CA, USA

Gösta Grahne Concordia University, Montreal, QC, Canada

Fabio Grandi Alma Mater Studiorum Università di Bologna, Bologna, Italy

Tyrone Grandison Proficiency Labs, Ashland, OR, USA

Peter M. D. Gray University of Aberdeen, Aberdeen, UK

Todd J. Green University of Pennsylvania, Philadelphia, PA, USA

Georges Grinstein University of Massachusetts, Lowell, MA, USA

Tom Gruber RealTravel, Emerald Hills, CA, USA

Le Gruenwald School of Computer Science, University of Oklahoma, Norman, OK, USA

Torsten Grust University of Tübingen, Tübingen, Germany

Dirk Van Gucht Indiana University, Bloomington, IN, USA

Carlos Guestrin Carnegie Mellon University, Pittsburgh, PA, USA

Dimitrios Gunopulos Department of Computer Science and Engineering, The University of California at Riverside, Bourns College of Engineering, Riverside, CA, USA

Amarnath Gupta San Diego Supercomputer Center, University of California San Diego, La Jolla, CA, USA

Himanshu Gupta Stony Brook University, Stony Brook, NY, USA

Cathal Gurrin Dublin City University, Dublin, Ireland

Ralf Hartmut Güting Fakultät für Mathematik und Informatik, Fernuniversität Hagen, Hagen, Germany

Computer Science, University of Hagen, Hagen, Germany 
Marc Gyssens Hasselt University, Hasselt, Belgium

Peter J. Haas IBM Almaden Research Center, San Jose, CA, USA

Karl Hahn BMW AG, Munich, Germany

Jean-Luc Hainaut University of Namur, Namur, Belgium

Alon Halevy The Recruit Institute of Technology, Mountain View, CA, USA Google Inc., Mountain View, CA, USA

Maria Halkidi University of Piraeus, Piraeus, Greece

Terry Halpin Neumont University, South Jordan, UT, USA

Jiawei Han University of Illinois at Urbana-Champaign, Urbana, IL, USA

Alan Hanjalic Delft University of Technology, Delft, The Netherlands

David Hansen The Australian e-Health Research Centre, Brisbane, QLD, Australia

Jörgen Hansson University of Skövde, Skövde, Sweden

Nikos Hardavellas Carnegie Mellon University, Pittsburgh, PA, USA

Theo Härder University of Kaiserslautern, Kaiserslautern, Germany

David Harel The Weizmann Institute of Science, Rehovot, Israel

Jayant R. Haritsa Indian Institute of Science, Bangalore, India

Stavros Harizopoulos HP Labs, Palo Alto, CA, USA

Per F. V. Hasle Royal School of Library and Information Science, University of Copenhagen, Copenhagen S, Denmark

Jordan T. Hastings Department of Geography, University of CaliforniaSanta Barbara, Santa Barbara, CA, USA

Alexander Hauptmann Carnegie Mellon University, Pittsburgh, PA, USA

Helwig Hauser University of Bergen, Bergen, Norway

Manfred Hauswirth Open Distributed Systems, Technical University of Berlin, Berlin, Germany

Fraunhofer FOKUS, Galway, Germany

Ben He University of Glasgow, Glasgow, UK

Thomas Heinis Imperial College London, London, UK

Pat Helland Microsoft Corporation, Redmond, WA, USA

Joseph M. Hellerstein University of California-Berkeley, Berkeley, CA, USA

Jean Henrard University of Namur, Namur, Belgium

John Herring Oracle USA Inc, Nashua, NH, USA 
Nicolas Hervé INRIA Paris-Rocquencourt, Le Chesnay, France

Marcus Herzog Vienna University of Technology, Vienna, Austria

Jean-Marc Hick University of Namur, Namur, Belgium

Jan Hidders University of Antwerp, Antwerpen, Belgium

Djoerd Hiemstra University of Twente, Enschede, The Netherlands

Linda L. Hill University of California-Santa Barbara, Santa Barbara, CA, USA

Alexander Hinneburg Institute of Computer Science, Martin-LutherUniversity Halle-Wittenberg, Halle/Saale, Germany

Hans Hinterberger Department of Computer Science, ETH Zurich, Zurich, Switzerland

Howard Ho IBM Almaden Research Center, San Jose, CA, USA

Erik Hoel Environmental Systems Research Institute, Redlands, CA, USA

Vasant Honavar Iowa State University, Ames, IA, USA

Mingsheng Hong Cornell University, Ithaca, NY, USA

Katja Hose Department of Computer Science, Aalborg University, Aalborg, Denmark

Haruo Hosoya The University of Tokyo, Tokyo, Japan

Vagelis Hristidis Department of Computer Science and Engineering, University of California, Riverside, Riverside, CA, USA

Wynne Hsu National University of Singapore, Singapore, Singapore

Yu-Ling Hsueh Computer Science and Information Engineering Department, National Chung Cheng University, Taiwan, Republic of China

Jian Hu Microsoft Research Asia, Haidian, China

Kien A. Hua University of Central Florida, Orlando, FL, USA

Xian-Sheng Hua Microsoft Research Asia, Beijing, China

Jun Huan University of Kansas, Lawrence, KS, USA

Haoda Huang Microsoft Research Asia, Beijing, China

Michael Huggett University of British Columbia, Vancouver, BC, Canada

Patrick C. K. Hung University of Ontario Institute of Technology, Oshawa, ON, Canada

Jeong-Hyon Hwang Department of Computer Science, University at Albany - State University of New York, Albany, NY, USA

Noha Ibrahim Grenoble Informatics Laboratory (LIG), Grenoble, France 
Ichiro Ide Graduate School of Informatics, Nagoya University, Nagoya, Aichi, Japan

Sergio Ilarri University of Zaragoza, Zaragoza, Spain

Ihab F. Ilyas Cheriton School of Computer Science, University of Waterloo, Waterloo, ON, Canada

Alfred Inselberg Tel Aviv University, Tel Aviv, Israel

Yannis Ioannidis University of Athens, Athens, Greece

Ekaterini Ioannou Faculty of Pure and Applied Sciences, Open University of Cyprus, Nicosia, Cyprus

Panagiotis G. Ipeirotis New York University, New York, NY, USA

Zachary G. Ives Computer and Information Science Department, University of Pennsylvania, Philadelphia, PA, USA

Hans-Arno Jacobsen Department of Electrical and Computer Engineering, University of Toronto, Toronto, ON, Canada

H. V. Jagadish University of Michigan, Ann Arbor, MI, USA

Alejandro Jaimes Telefonica R\&D, Madrid, Spain

Ramesh Jain University of California, Irvine, CA, USA

Sushil Jajodia George Mason University, Fairfax, VA, USA

Greg Janée University of California-Santa Barbara, Santa Barbara, CA, USA

Kalervo Järvelin University of Tampere, Tampere, Finland

Christian S. Jensen Department of Computer Science, Aalborg University, Aalborg, Denmark

Eric C. Jensen Twitter, Inc., San Francisco, CA, USA

Manfred Jeusfeld IIT, University of Skövde, Skövde, Sweden

Aura Frames, New York City, NY, USA

Heng Ji New York University, New York, NY, USA

Zhe Jiang University of Alabama, Tuscaloosa, AL, USA

Ricardo Jiménez-Peris Distributed Systems Lab, Universidad Politecnica de Madrid, Madrid, Spain

Hai Jin Service Computing Technology and System Lab, Cluster and Grid Computing Lab, School of Computer Science and Technology, Huazhong University of Science and Technology, Wuhan, China

Jiashun Jin Carnegie Mellon University, Pittsburgh, PA, USA

Ruoming Jin Department of Computer Science, Kent State University, Kent, OH, USA 
Ryan Johnson Carnegie Mellon University, Pittsburg, PA, USA

Theodore Johnson AT\&T Labs - Research, Florham Park, NJ, USA

Christopher B. Jones Cardiff University, Cardiff, UK

Rosie Jones Yahoo! Research, Burbank, CA, USA

James B. D. Joshi University of Pittsburgh, Pittsburgh, PA, USA

Vanja Josifovski Uppsala University, Uppsala, Sweden

Marko Junkkari University of Tampere, Tampere, Finland

Jan Jurjens The Open University, Buckinghamshire, UK

Mouna Kacimi Max-Planck Institute for Informatics, Saarbrücken, Germany

Tamer Kahveci University of Florida, Gainesville, FL, USA

Panos Kalnis National University of Singapore, Singapore, Singapore

Jaap Kamps University of Amsterdam, Amsterdam, The Netherlands

James Kang University of Minnesota, Minneapolis, MN, USA

Carl-Christian Kanne University of Mannheim, Mannheim, Germany

Aman Kansal Microsoft Research, Redmond, WA, USA

Murat Kantarcioğlu University of Texas at Dallas, Richardson, TX, USA

Ben Kao Department of Computer Science, The University of Hong Kong, Hong Kong, China

George Karabatis University of Maryland, Baltimore Country (UMBC), Baltimore, MD, USA

Grigoris Karvounarakis LogicBlox, Atlanta, GA, USA

George Karypis University of Minnesota, Minneapolis, MN, USA

Vipul Kashyap Clinical Programs, CIGNA Healthcare, Bloomfield, CT, USA

Yannis Katsis University of California-San Diego, La Jolla, CA, USA

Raghav Kaushik Microsoft Research, Redmond, WA, USA

Gabriella Kazai Microsoft Research Cambridge, Cambridge, UK

Daniel A. Keim Computer Science Department, University of Konstanz, Konstanz, Germany

Jaana Kekäläinen University of Tampere, Tampere, Finland

Anastasios Kementsietsidis IBM T.J. Watson Research Center, Hawthorne, NY, USA

Bettina Kemme School of Computer Science, McGill University, Montreal, QC, Canada 
Jessie Kennedy Napier University, Edinburgh, UK

Vijay Khatri Operations and Decision Technologies Department, Kelley School of Business, Indiana University, Bloomington, IN, USA

Ashfaq Khokhar University of Illinois at Chicago, Chicago, IL, USA

Daniel Kifer Yahoo! Research, Santa Clara, CA, USA

Stephen Kimani Director ICSIT, Jomo Kenyatta University of Agriculture and Technology (JKUAT), Juja, Kenya

Sofia Kleisarchaki CNRS, Univ. Grenoble Alps, Grenoble, France

Craig A. Knoblock University of Southern California, Marina del Rey, Los Angeles, CA, USA

Christoph Koch Cornell University, Ithaca, New York, NY, USA

EPFL, Lausanne, Switzerland

Solmaz Kolahi University of British Columbia, Vancouver, BC, Canada

George Kollios Boston University, Boston, MA, USA

Christian Koncilia Institute of Informatics-Systems, University of Klagenfurt, Klagenfurt, Austria

Roberto Konow Department of Computer Science, University of Chile, Santiago, Chile

Marijn Koolen Research and Development, Huygens ING, Royal Netherlands Academy of Arts and Sciences, Amsterdam, The Netherlands

David Koop University of Massachusetts Dartmouth, Dartmouth, MA, USA

Poon Wei Koot Nanyang Technological University, Singapore, Singapore

Julius Köpke Department of Informatics-Systems, Alpen-Adria-Universität Klagenfurt, Klagenfurt, Austria

Flip R. Korn AT\&T Labs-Research, Florham Park, NJ, USA

Harald Kosch University of Passau, Passau, Germany

Cartik R. Kothari Biomedical Informatics, Ohio State University, College of Medicine, Columbus, OH, USA

Yannis Kotidis Department of Informatics, Athens University of Economics and Business, Athens, Greece

Spyros Kotoulas VU University Amsterdam, Amsterdam, The Netherlands Manolis Koubarakis University of Athens, Athens, Greece

Konstantinos Koutroumbas Institute for Space Applications and Remote Sensing, Athens, Greece

Bernd J. Krämer University of Hagen, Hagen, Germany 
Tim Kraska Department of Computer Science, Brown University, Providence, RI, USA

Werner Kriechbaum IBM Development Lab, Böblingen, Germany

Hans-Peter Kriegel Ludwig-Maximilians-University, Munich, Germany

Chandra Krintz Department of Computer Science, University of California, Santa Barbara, CA, USA

Rajasekar Krishnamurthy IBM Almaden Research Center, San Jose, CA, USA

Peer Kröger Ludwig-Maximilians-Universität München, Munich, Germany

Thomas Kühne School of Engineering and Computer Science, Victoria University of Wellington, Wellington, New Zealand

Krishna Kulkarni Independent Consultant, San Jose, CA, USA

Ravi Kumar Yahoo Research, Santa Clara, CA, USA

Nicholas Kushmerick VMWare, Seattle, WA, USA

Alan G. Labouseur School of Computer Science and Mathematics, Marist College, Poughkeepsie, NY, USA

Alexandros Labrinidis Department of Computer Science, University of Pittsburgh, Pittsburgh, PA, USA

Zoé Lacroix Arizona State University, Tempe, AZ, USA

Alberto H. F. Laender Federal University of Minas Gerais, Belo Horizonte, Brazil

Bibudh Lahiri Iowa State University, Ames, IA, USA

Laks V. S. Lakshmanan University of British Columbia, Vancouver, BC, Canada

Mounia Lalmas Yahoo! Inc., London, UK

Lea Landucci University of Florence, Florence, Italy

Birger Larsen Royal School of Library and Information Science, Copenhagen, Denmark

Mary Lynette Larsgaard University of California-Santa Barbara, Santa Barbara, CA, USA

Per-Åke Larson Microsoft Corporation, Redmond, WA, USA

Robert Laurini INSA-Lyon, University of Lyon, Lyon, France

LIRIS, INSA-Lyon, Lyon, France

Georg Lausen University of Freiburg, Freiburg, Germany

Jens Lechtenbörger University of Münster, Münster, Germany 
Thierry Lecroq Université de Rouen, Rouen, France

Dongwon Lee The Pennsylvania State University, Park, PA, USA

Victor E. Lee John Carroll University, University Heights, OH, USA

Yang W. Lee College of Business Administration, Northeastern University, Boston, MA, USA

Pieter De Leenheer Vrije Universiteit Brussel, Collibra NV, Brussels, Belgium

Wolfgang Lehner Dresden University of Technology, Dresden, Germany

Domenico Lembo Dip. di Ingegneria Informatica Automatica e Gestionale Antonio Ruberti, Sapienza Università di Roma, Rome, Italy

Ronny Lempel Yahoo! Research, Haifa, Israel

Maurizio Lenzerini Dip. di Ingegneria Informatica Automatica e Gestionale Antonio Ruberti, Sapienza Università di Roma, Rome, Italy

Kristina Lerman University of Southern California, Marina del Rey, Los Angeles, CA, USA

Ulf Leser Humboldt University of Berlin, Berlin, Germany

Carson Kai-Sang Leung Department of Computer Science, University of Manitoba, Winnipeg, MB, Canada

Mariano Leva Dipartimento di Ingegneria Informatica, Automatica e Gestionale “A.Ruberti”, Sapienza - Università di Roma, Roma, Italy

Stefano Levialdi Sapienza University of Rome, Rome, Italy

Brian Levine University of Massachusetts, Amherst, MA, USA

Changqing Li Duke University, Durham, NC, USA

Chen Li University of California - Irvine, School of Information and Computer Sciences, Irvine, CA, USA

Chengkai Li University of Texas at Arlington, Arlington, TX, USA

Hua Li Microsoft Research Asia, Beijing, China

Jinyan Li Nanyang Technological University, Singapore, Singapore

Ninghui Li Purdue University, West Lafayette, IN, USA

Ping Li Cornell University, Ithaca, NY, USA

Qing Li City University of Hong Kong, Hong Kong, China

Xue Li The University of Queensland, Brisbane, QLD, Australia

Yunyao Li IBM Almaden Research Center, San Jose, CA, USA

Ying Li Cognitive People Solutions, IBM Human Resources, Armonk, NY, USA 
Xiang Lian Department of Computer Science, Kent State University, Kent, $\mathrm{OH}, \mathrm{USA}$

Leonid Libkin School of Informatics, University of Edinburgh, Edinburgh, Scotland, UK

Sam S. Lightstone IBM Canada Ltd, Markham, ON, Canada

Jimmy Lin University of Maryland, College Park, MD, USA

Tsau Young Lin Department of Computer Science, San Jose State University, San Jose, CA, USA

Xuemin Lin University of New South Wales, Sydney, NSW, Australia

Tok Wang Ling National University of Singapore, Singapore, Singapore

Bing Liu University of Illinois at Chicago, Chicago, IL, USA

Danzhou Liu University of Central Florida, Orlando, FL, USA

Guimei Liu Institute for Infocomm Research, Singapore, Singapore

Huan Liu Data Mining and Machine Learning Lab, School of Computing, Informatics, and Decision Systems Engineering, Arizona State University, Tempe, AZ, USA

Jinze Liu University of Kentucky, Lexington, KY, USA

Lin Liu Department of Computer Science, Kent State University, Kent, OH, USA

Ning Liu Microsoft Research Asia, Beijing, China

Qing Liu CSIRO, Hobart, TAS, Australia

Xiangyu Liu Xiamen University, Xiamen, China

Vebjorn Ljosa Broad Institute of MIT and Harvard, Cambridge, MA, USA

David Lomet Microsoft Research, Redmond, WA, USA

Cheng Long School of Electronics, Electrical Engineering and Computer Science, Queen's University Belfast, Kowloon, Hong Kong

Boon Thau Loo ETH Zurich, Zurich, Switzerland

Phillip Lord Newcastle University, Newcastle-Upon-Tyne, UK

Nikos A. Lorentzos Informatics Laboratory, Department of Agricultural Economics and Rural Development, Agricultural University of Athens, Athens, Greece

Lie Lu Microsoft Research Asia, Beijing, China

Bertram Ludäscher University of California-Davis, Davis, CA, USA

Yan Luo University of Illinois at Chicago, Chicago, IL, USA

Yves A. Lussier University of Chicago, Chicago, IL, USA 
Ioanna Lykourentzou CRP Henri Tudor, Esch-sur-Alzette, Luxembourg

Craig MacDonald University of Glasgow, Glasgow, UK

Ashwin Machanavajjhala Cornell University, Ithaca, NY, USA

Samuel Madden Massachusetts Institute of Technology, Cambridge, MA, USA

Paola Magillo University of Genova, Genoa, Italy

Ahmed R. Mahmood Computer Science, Purdue University, West Lafayette, IN, USA

David Maier Portland State University, Portland, OR, USA

Ratul kr. Majumdar Department of Computer Science and Engineering, Indian Institute of Technology Bombay, Mumbai, India

Jan Małuszyński Linköping University, Linköping, Sweden

Nikos Mamoulis University of Hong Kong, Hong Kong, China

Stefan Manegold CWI, Amsterdam, The Netherlands

Murali Mani Worcester Polytechnic, Worcester, MA, USA

Serge Mankovski CA Labs, CA Inc., Thornhill, ON, Canada

Ioana Manolescu INRIA Saclay-Îlle de France, Orsay, France

Yannis Manolopoulos Aristotle University of Thessaloniki, Thessaloniki, Greece

Florian Mansmann University of Konstanz, Konstanz, Germany

Svetlana Mansmann University of Konstanz, Konstanz, Germany

Shahar Maoz The Weizmann Institute of Science, Rehovot, Israel

Patrick Marcel Département Informatique, Laboratoire d'Informatique, Université François Rabelais Tours, Blois, France

Amélie Marian Computer Science Department, Rutgers University, New Brunswick, NJ, USA

Volker Markl IBM Almaden Research Center, San Jose, CA, USA

David Martin Nuance Communications, Sunnyvale, CA, USA

Maria Vanina Martinez University of Maryland, College Park, MD, USA

Maristella Matera Politecnico di Milano, Milan, Italy

Michael Mathioudakis Université de Lyon, CNRS, INSA-Lyon, LIRIS, UMR5205, F-69621, France

Marta Mattoso Federal University of Rio de Janeiro, Rio de Janeiro, Brazil Andrea Maurino University of Milano-Bicocca, Milan, Italy 
Jose-Norberto Mazón University of Alicante, Alicante, Spain

John McCloud CERT Insider Threat Center, Software Engineering Institute, Carnegie Mellon University, Pittsburgh, PA, USA

Kevin S. McCurley Google Research, Mountain View, CA, USA

Andrew McGregor Microsoft Research, Silicon Valley, Mountain View, CA, USA

Timothy McPhillips University of California-Davis, Davis, CA, USA

Massimo Mecella Dipartimento di Ingegneria Informatica, Automatica e Gestionale “A.Ruberti”, Sapienza - Università di Roma, Roma, Italy

Brahim Medjahed The University of Michigan-Dearborn, Dearborn, MI, USA

Carlo Meghini The Italian National Research Council, Pisa, Italy

Tao Mei Microsoft Research Asia, Beijing, China

Jonas Mellin University of Skövde, The Informatics Research Centre, Skövde, Sweden

University of Skövde, School of Informatics, Skövde, Sweden

Massimo Melucci University of Padua, Padua, Italy

Niccolò Meneghetti Computer Science and Engineering Department, University at Buffalo, Buffalo, NY, USA

Weiyi Meng Department of Computer Science, State University of New York at Binghamton, Binghamton, NY, USA

Ahmed Metwally LinkedIn Corp., Mountain View, CA, USA

Jan Michels Oracle Corporation, Redwood Shores, CA, USA

Gerome Miklau University of Massachusetts, Amherst, MA, USA

Alessandra Mileo Insight Centre for Data Analytics, Dublin City University, Dublin, Ireland

Harvey J. Miller University of Utah, Salt Lake City, UT, USA

Renée J. Miller Department of Computer Science, University of Toronto, Toronto, ON, Canada

Tova Milo School of Computer Science, Tel Aviv University, Tel Aviv, Israel Umar Farooq Minhas Microsoft Research, Redmond, WA, USA

Paolo Missier School of Computing Science, Newcastle University, Newcastle upon Tyne, UK

Prasenjit Mitra The Pennsylvania State University, University Park, PA, USA

Michael Mitzenmacher Harvard University, Boston, MA, USA 
Mukesh Mohania IBM Research, Melbourne, VIC, Australia

Mohamed F. Mokbel Department of Computer Science and Engineering, University of Minnesota-Twin Cities, Minneapolis, MN, USA

Angelo Montanari University of Udine, Udine, Italy

Reagan W. Moore School of Information and Library Science, University of North Carolina at Chapel Hill, Chapel Hill, NC, USA

Konstantinos Morfonios Oracle, Redwood City, CA, USA

Peter Mork The MITRE Corporation, McLean, VA, USA

Mirella M. Moro Departamento de Ciencia da Computaçao, Universidade Federal de Minas Gerais - UFMG, Belo Horizonte, MG, Brazil

Kyriakos Mouratidis Singapore Management University, Singapore, Singapore

Kamesh Munagala Duke University, Durham, NC, USA

Ethan V. Munson Department of EECS, University of WisconsinMilwaukee, Milwaukee, WI, USA

Shawn Murphy Massachusetts General Hospital, Boston, MA, USA

John Mylopoulos Department of Computer Science, University of Toronto, Toronto, ON, Canada

Marta Patiño-Martínez Distributed Systems Lab, Universidad Politecnica de Madrid, Madrid, Spain

ETSI Informáticos, Universidad Politécnica de Madrid (UPM), Madrid, Spain

Frank Nack University of Amsterdam, Amsterdam, The Netherlands

Marc Najork Google, Inc., Mountain View, CA, USA

Ullas Nambiar Zensar Technologies Ltd, Pune, India

Alexandros Nanopoulos Aristotle University, Thessaloniki, Greece

Vivek Narasayya Microsoft Corporation, Redmond, WA, USA

Mario A. Nascimento Department of Computing Science, University of Alberta, Edmonton, AB, Canada

Alan Nash Aleph One LLC, La Jolla, CA, USA

Harald Naumann Vienna University of Technology, Vienna, Austria

Gonzalo Navarro Department of Computer Science, University of Chile, Santiago, Chile

Wolfgang Nejdl L3S Research Center, University of Hannover, Hannover, Germany

Thomas Neumann Max-Planck Institute for Informatics, Saarbrücken, Germany 
Bernd Neumayr Department for Business Informatics - Data and Knowledge Engineering, Johannes Kepler University Linz, Linz, Austria

Frank Neven Hasselt University and Transnational University of Limburg, Diepenbeek, Belgium

Chong-Wah Ngo City University of Hong Kong, Hong Kong, China

Peter Niblett IBM United Kingdom Limited, Winchester, UK

Naoko Nitta Osaka University, Osaka, Japan

Igor Nitto Department of Computer Science, University of Pisa, Pisa, Italy

Cheng Niu Microsoft Research Asia, Beijing, China

Vilém Novák Institute for Research and Applications of Fuzzy Modeling, University of Ostrava, Ostrava, Czech Republic

Chimezie Ogbuji Cleveland Clinic Foundation, Cleveland, OH, USA

Peter Øhrstrøm Aalborg University, Aalborg, Denmark

Christine M. O'Keefe CSIRO Preventative Health National Research Flagship, Acton, ACT, Australia

Paul W. Olsen Department of Computer Science, The College of Saint Rose, Albany, NY, USA

Dan Olteanu Department of Computer Science, University of Oxford, Oxford, UK

Behrooz Omidvar-Tehrani Interactive Data Systems Group, Ohio State University, Columbus, OH, USA

Patrick O'Neil University of Massachusetts, Boston, MA, USA

Beng Chin Ooi School of Computing, National University of Singapore, Singapore, Singapore

Iadh Ounis University of Glasgow, Glasgow, UK

Mourad Ouzzani Qatar Computing Research Institute, HBKU, Doha, Qatar

Fatma Özcan IBM Research - Almaden, San Jose, CA, USA

M. Tamer Özsu Cheriton School of Computer Science, University of Waterloo, Waterloo, ON, Canada

Esther Pacitti INRIA and LINA, University of Nantes, Nantes, France

Chris D. Paice Lancaster University, Lancaster, UK

Noël de Palma INPG - INRIA, Grenoble, France

Nathaniel Palmer Workflow Management Coalition, Hingham, MA, USA

Themis Palpanas Paris Descartes University, Paris, France

Biswanath Panda Cornell University, Ithaca, NY, USA 
Ippokratis Pandis Carnegie Mellon University, Pittsburgh, PA, USA

Amazon Web Services, Seattle, WA, USA

Dimitris Papadias Department of Computer Science and Engineering, Hong Kong University of Science and Technology, Kowloon, Hong Kong, Hong Kong

Spiros Papadimitriou IBM T.J. Watson Research Center, Hawthorne, NY, USA

Apostolos N. Papadopoulos Aristotle University of Thessaloniki, Thessaloniki, Greece

Yannis Papakonstantinou University of California-San Diego, La Jolla, CA, USA

Jan Paredaens University of Antwerp, Antwerpen, Belgium

Christine Parent University of Lausanne, Lausanne, Switzerland

Josiane Xavier Parreira Siemens AG, Galway, Austria

Gabriella Pasi Department of Informatics, Systems and Communication, University of Milano-Bicocca, Milan, Italy

Chintan Patel Columbia University, New York, NY, USA

Jignesh M. Patel University of Wisconsin-Madison, Madison, WI, USA

Norman W. Paton University of Manchester, Manchester, UK

Cesare Pautasso University of Lugano, Lugano, Switzerland

Torben Bach Pedersen Department of Computer Science, Aalborg University, Aalborg, Denmark

Fernando Pedone Università della Svizzera Italiana (USI), Lugano, Switzerland

Jovan Pehcevski INRIA Paris-Rocquencourt, Le Chesnay Cedex, France

Jian Pei School of Computing Science, Simon Fraser University, Burnaby, BC, Canada

Ronald Peikert ETH Zurich, Zurich, Switzerland

Mor Peleg Department of Information Systems, University of Haifa, Haifa, Israel

Fuchun Peng Yahoo! Inc., Sunnyvale, CA, USA

Peng Peng Alibaba, Yu Hang District, Hangzhou, China

Liam Peyton University of Ottawa, Ottawa, ON, Canada

Dieter Pfoser Department of Geography and Geoinformation Science, George Mason University, Fairfax, VA, USA 
Danh Le Phuoc Open Distributed Systems, Technical University of Berlin, Berlin, Germany

Mario Piattini University of Castilla-La Mancha, Ciudad Real, Spain

Benjamin C. Pierce University of Pennsylvania, Philadelphia, PA, USA

Karen Pinel-Sauvagnat IRIT laboratory, University of Toulouse, Toulouse, France

Leo L. Pipino University of Massachusetts, Lowell, MA, USA

Peter Pirolli Palo Alto Research Center, Palo Alto, CA, USA

Evaggelia Pitoura Department of Computer Science and Engineering, University of Ioannina, Ioannina, Greece

Benjamin Piwowarski University of Glasgow, Glasgow, UK

Vassilis Plachouras Yahoo! Research, Barcelona, Spain

Catherine Plaisant University of Maryland, College Park, MD, USA

Claudia Plant University of Vienna, Vienna, Austria

Christian Platzer Technical University of Vienna, Vienna, Austria

Dimitris Plexousakis Foundation for Research and Technology-Hellas (FORTH), Heraklion, Greece

Neoklis Polyzotis University of California Santa Cruz, Santa Cruz, CA, USA

Raymond K. Pon University of California, Los Angeles, CA, USA

Lucian Popa IBM Almaden Research Center, San Jose, CA, USA

Alexandra Poulovassilis University of London, London, UK

Sunil Prabhakar Purdue University, West Lafayette, IN, USA

Cecilia M. Procopiuc AT\&T Labs, Florham Park, NJ, USA

Enrico Puppo Department of Informatics, Bioengineering, Robotics and Systems Engineering, University of Genova, Genoa, Italy

Ross S. Purves University of Zurich, Zurich, Switzerland

Vivien Quéma CNRS, INRIA, Saint-Ismier Cedex, France

Christoph Quix RWTH Aachen University, Aachen, Germany

Sriram Raghavan IBM Almaden Research Center, San Jose, CA, USA

Erhard Rahm University of Leipzig, Leipzig, Germany

Habibur Rahman Department of Computer Science and Engineering, University of Texas at Arlington, Arlington, TX, USA

Krithi Ramamritham Department of Computer Science and Engineering, Indian Institute of Technology Bombay, Mumbai, India 
Maya Ramanath Max-Planck Institute for Informatics, Saarbrücken, Germany

Georgina Ramírez Yahoo! Research Barcelona, Barcelona, Spain

Edie Rasmussen Library, Archival and Information Studies, The University of British Columbia, Vancouver, BC, Canada

Indrakshi Ray Colorado State University, Fort Collins, CO, USA

Colin R. Reeves Coventry University, Coventry, UK

Payam Refaeilzadeh Google Inc., Los Angeles, CA, USA

D. R. Reforgiato University of Maryland, College Park, MD, USA

Bernd Reiner Technical University of Munich, Munich, Germany

Frederick Reiss IBM Almaden Research Center, San Jose, CA, USA

Harald Reiterer University of Konstanz, Constance, Germany

Matthias Renz Ludwig-Maximilians-Universität München, Munich, Germany

Andreas Reuter Heidelberg Laureate Forum Foundation, SchlossWolfsbrunnenweg 33, Heidelberg, Germany

Peter Revesz University of Nebraska-Lincoln, Lincoln, NE, USA

Mirek Riedewald Cornell University, Ithaca, NY, USA

Rami Rifaieh University of California-San Diego, San Diego, CA, USA

Stefanie Rinderle-Ma University of Vienna, Vienna, Austria

Tore Risch Department of Information Technology, Uppsala University, Uppsala, Sweden

Thomas Rist University of Applied Sciences, Augsburg, Germany

Stefano Rizzi DISI, University of Bologna, Bologna, Italy

Stephen Robertson Microsoft Research Cambridge, Cambridge, UK

Roberto A. Rocha Partners eCare, Partners HealthCare System, Wellesley, MA, USA

John F. Roddick Flinders University, Adelaide, SA, Australia

Thomas Roelleke Queen Mary University of London, London, UK

Didier Roland University of Namur, Namur, Belgium

Oscar Romero Polytechnic University of Catalonia, Barcelona, Spain

Rafael Romero University of Alicante, Alicante, Spain

Riccardo Rosati Dip. di Ingegneria Informatica Automatica e Gestionale Antonio Ruberti, Sapienza Università di Roma, Rome, Italy 
Timothy Roscoe ETH Zurich, Zurich, Switzerland

Kenneth A. Ross Columbia University, New York, NY, USA

Prasan Roy Sclera, Inc., Walnut, CA, USA

Senjuti Basu Roy Department of Computer Science, New Jersey Institute of Technology, Tacoma, WA, USA

Sudeepa Roy Department of Computer Science, Duke University, Durham, NC, USA

Yong Rui Microsoft China R\&D Group, Redmond, WA, USA

Dan Russler Oracle Health Sciences, Redwood Shores, CA, USA

Georgia Tech Research Institute, Atlanta, Georgia, USA

Michael Rys Microsoft Corporation, Sammamish, WA, USA

Giovanni Maria Sacco Dipartimento di Informatica, Università di Torino, Torino, Italy

Tetsuya Sakai Waseda University, Tokyo, Japan

Kenneth Salem University of Waterloo, Waterloo, ON, Canada

Simonas Šaltenis Aalborg University, Aalborg, Denmark

George Samaras University of Cyprus, Nicosia, Cyprus

Giuseppe Santucci University of Rome, Rome, Italy

Maria Luisa Sapino University of Turin, Turin, Italy

Sunita Sarawagi IIT Bombay, Mumbai, India

Anatol Sargin University of Augsburg, Augsburg, Germany

Mohamed Sarwat School of Computing, Informatics, and Decision Systems Engineering, Arizona State University, Tempe, AZ, USA

Kai-Uwe Sattler Technische Universität Ilmenau, Ilmenau, Germany

Monica Scannapieco University of Rome, Rome, Italy

Matthias Schäfer University of Konstanz, Konstanz, Germany

Sebastian Schaffert Salzburg Research, Salzburg, Austria

Ralf Schenkel Campus II Department IV - Computer Science, Professorship for databases and information systems, University of Trier, Trier, Germany

Raimondo Schettini University of Milano-Bicocca, Milan, Italy

Peter Scheuermann Department of ECpE, Iowa State University, Ames, IA, USA

Ulrich Schiel Federal University of Campina Grande, Campina Grande, Brazil 
Markus Schneider University of Florida, Gainesville, FL, USA

Marc H. Scholl University of Konstanz, Konstanz, Germany

Michel Scholl Cedric-CNAM, Paris, France

Tobias Schreck Department of Computer Science and Biomedical Engineering, Institute of Computer Graphics and Knowledge Visualization, Graz University of Technology, Graz, Austria

Michael Schrefl University of Linz, Linz, Austria

Erich Schubert Heidelberg University, Heidelberg, Germany

Matthias Schubert Ludwig-Maximilians-University, Munich, Germany

Christoph G. Schuetz Department for Business Informatics - Data and Knowledge Engineering, Johannes Kepler University Linz, Linz, Austria

Heiko Schuldt Department of Mathematics and Computer Science, Databases and Information Systems Research Group, University of Basel, Basel, Switzerland

Heidrun Schumann University of Rostock, Rostock, Germany

Felix Schwagereit University of Koblenz-Landau, Koblenz, Germany

Nicole Schweikardt Johann Wolfgang Goethe-University, Frankfurt am Main, Frankfurt, Germany

Fabrizio Sebastiani Qatar Computing Research Institute, Doha, Qatar

Nicu Sebe University of Amsterdam, Amsterdam, Netherlands

Monica Sebillo University of Salerno, Salerno, Italy

Thomas Seidl RWTH Aachen University, Aachen, Germany

Manuel Serrano University of Alicante, Alicante, Spain

Amnon Shabo (Shvo) University of Haifa, Haifa, Israel

Mehul A. Shah Amazon Web Services (AWS), Seattle, WA, USA

Nigam Shah Stanford University, Stanford, CA, USA

Cyrus Shahabi University of Southern California, Los Angeles, CA, USA Jayavel Shanmugasundaram Yahoo Research!, Santa Clara, NY, USA

Marc Shapiro Inria Paris, Paris, France

Sorbonne-Universités-UPMC-LIP6, Paris, France

Mohamed Sharaf Electrical and Computer Engineering, University of Toronto, Toronto, ON, Canada

Mehdi Sharifzadeh Google, Santa Monica, CA, USA

Jayant Sharma Oracle USA Inc, Nashua, NH, USA

Guy Sharon IBM Research Labs-Haifa, Haifa, Israel 
Dennis Shasha Department of Computer Science, New York University, New York, NY, USA

Shashi Shekhar Department of Computer Science, University of Minnesota, Minneapolis, MN, USA

Jialie Shen Singapore Management University, Singapore, Singapore

Xuehua Shen Google, Inc., Mountain View, CA, USA

Dou Shen Microsoft Corporation, Redmond, WA, USA

Baidu, Inc., Beijing City, China

Heng Tao Shen School of Information Technology and Electrical Engineering, The University of Queensland, Brisbane, QLD, Australia

University of Electronic Science and Technology of China, Chengdu, Sichuan Sheng, China

Rao Shen Yahoo!, Sunnyvale, CA, USA

Frank Y. Shih New Jersey Institute of Technology, Newark, NJ, USA

Arie Shoshani Lawrence Berkeley National Laboratory, Berkeley, CA, USA

Pavel Shvaiko University of Trento, Trento, Italy

Wolf Siberski L3S Research Center, University of Hannover, Hannover, Germany

Ronny Siebes VU University Amsterdam, Amsterdam, The Netherlands

Laurynas Šikšnys Department of Computer Science, Aalborg University, Aalborg, Denmark

Adam Silberstein Yahoo! Research Silicon Valley, Santa Clara, CA, USA

Fabrizio Silvestri Yahoo Inc, London, UK

Alkis Simitsis HP Labs, Palo Alto, CA, USA

Simeon J. Simoff University of Western Sydney, Sydney, NSW, Australia

Elena Simperl Electronics and Computer Science, University of Southampton, Southampton, UK

Radu Sion Stony Brook University, Stony Brook, NY, USA

Mike Sips Stanford University, Stanford, CA, USA

Cristina Sirangelo IRIF, Paris Diderot University, Paris, France

Yannis Sismanis IBM Almaden Research Center, Almaden, CA, USA

Hala Skaf-Molli Computer Science, University of Nantes, Nantes, France

Spiros Skiadopoulos University of Peloponnese, Tripoli, Greece 
Richard T. Snodgrass Department of Computer Science, University of Arizona, Tucson, AZ, USA

Dataware Ventures, Tucson, AZ, USA

Cees Snoek University of Amsterdam, Amsterdam, The Netherlands

Mohamed A. Soliman Datometry Inc., San Francisco, CA, USA

Il-Yeol Song College of Computing and Informatics, Drexel University, Philadelphia, PA, USA

Ruihua Song Microsoft Research Asia, Beijing, China

Jingkuan Song Columbia University, New York, NY, USA

Stefano Spaccapietra EPFL, Lausanne, Switzerland

Greg Speegle Department of Computer Science, Baylor University, Waco, TX, USA

Padmini Srinivasan The University of Iowa, Iowa City, IA, USA

Venkat Srinivasan Virginia Tech, Blacksburg, VA, USA

Divesh Srivastava AT\&T Labs - Research, AT\&T, Bedminster, NJ, USA

Steffen Staab Institute for Web Science and Technologies - WeST, University of Koblenz-Landau, Koblenz, Germany

Constantine Stephanidis Foundation for Research and Technology-Hellas (FORTH), Heraklion, Greece

University of Crete, Heraklion, Greece

Robert Stevens University of Manchester, Manchester, UK

Andreas Stoffel University of Konstanz, Konstanz, Germany

Michael Stonebraker Massachusetts Institute of Technology, Cambridge, MA, USA

Umberto Straccia The Italian National Research Council, Pisa, Italy

Martin J. Strauss University of Michigan, Ann Arbor, MI, USA

Diane M. Strong Worcester Polytechnic Institute, Worcester, MA, USA

Jianwen Su University of California-Santa Barbara, Santa Barbara, CA, USA

Kazimierz Subieta Polish-Japanese Institute of Information Technology, Warsaw, Poland

V.S. Subrahmanian University of Maryland, College Park, MD, USA

Dan Suciu University of Washington, Seattle, WA, USA 
S. Sudarshan Indian Institute of Technology, Bombay, India

Torsten Suel Yahoo! Research, Sunnyvale, CA, USA

Jian-Tao Sun Microsoft Research Asia, Beijing, China

Subhash Suri University of California-Santa Barbara, Santa Barbara, CA, USA

Jaroslaw Szlichta University of Ontario Institute of Technology, Oshawa, ON, Canada

Stefan Tai University of Karlsruhe, Karlsruhe, Germany

Kian-Lee Tan Department of Computer Science, National University of Singapore, Singapore, Singapore

Pang-Ning Tan Michigan State University, East Lansing, MI, USA

Wang-Chiew Tan University of California-Santa Cruz, Santa Cruz, CA, USA

Letizia Tanca Computer Science, Politecnico di Milano, Milan, Italy

Lei Tang Chief Data Scientist, Clari Inc., Sunnyvale, CA, USA

Wei Tang Teradata Corporation, El Segundo, CA, USA

Egemen Tanin Computing and Information Systems, University of Melbourne, Melbourne, VIC, Australia

Val Tannen Department of Computer and Information Science, University of Pennsylvania, Philadelphia, PA, USA

Abdullah Uz Tansel Baruch College, CUNY, New York, NY, USA

Yufei Tao Chinese University of Hong Kong, Hong Kong, China

Sandeep Tata IBM Almaden Research Center, San Jose, CA, USA

Nesime Tatbul Intel Labs and MIT, Cambridge, MA, USA

Christophe Taton INPG - INRIA, Grenoble, France

Behrooz Omidvar Tehrani Laboratoire d'Informatique de Grenoble, SaintMartin d'Hères, France

Paolo Terenziani Dipartimento di Scienze e Innovazione Tecnologica (DiSIT), Università del Piemonte Orientale "Amedeo Avogadro", Alessandria, Italy

Alexandre Termier LIG (Laboratoire d'Informatique de Grenoble), HADAS team, Université Joseph Fourier, Saint Martin d'Hères, France

Evimaria Terzi Computer Science Department, Boston University, Boston, MA, USA

IBM Almaden Research Center, San Jose, CA, USA

Bernhard Thalheim Christian-Albrechts University, Kiel, Germany 
Martin Theobald Institute of Databases and Information Systems (DBIS), Ulm University, Ulm, Germany

Stanford University, Stanford, CA, USA

Sergios Theodoridis University of Athens, Athens, Greece

Yannis Theodoridis University of Piraeus, Piraeus, Greece

Saravanan Thirumuruganathan Department of Computer Science and Engineering, University of Texas at Arlington, Arlington, TX, USA

Qatar Computing Research Institute, Hamad Bin Khalifa University, Doha, Qatar

Stephen W. Thomas Dataware Ventures, Kingston, ON, Canada

Alexander Thomasian Thomasian and Associates, Pleasantville, NY, USA

Christian Thomsen Department of Computer Science, Aalborg University, Aalborg, Denmark

Bhavani Thuraisingham The University of Texas at Dallas, Richardson, TX, USA

Srikanta Tirthapura Iowa State University, Ames, IA, USA

Wee Hyong Tok National University of Singapore, Singapore, Singapore

David Toman University of Waterloo, Waterloo, ON, Canada

Frank Tompa David R. Cheriton School of Computer Science, University of Waterloo, Waterloo, ON, Canada

Alejandro Z. Tomsic Sorbonne-Universités-UPMC-LIP6, Paris, France Inria Paris, Paris, France

Rodney Topor Griffith University, Nathan, Australia

Riccardo Torlone University of Rome, Rome, Italy

Kristian Torp Aalborg University, Aalborg, Denmark

Nicola Torpei University of Florence, Florence, Italy

Nerius Tradišauskas Aalborg University, Aalborg, Denmark

Goce Trajcevski Department of ECpE, Iowa State University, Ames, IA, USA

Peter Triantafillou University of Patras, Rio, Patras, Greece

Silke TrißI Humboldt University of Berlin, Berlin, Germany

Andrew Trotman University of Otago, Dunedin, New Zealand

Juan Trujillo Lucentia Research Group, Department of Information Languages and Systems, Facultad de Informática, University of Alicante, Alicante, Spain 
Beth Trushkowsky Department of Computer Science, Harvey Mudd College, Claremont, CA, USA

Panayiotis Tsaparas Department of Computer Science and Engineering, University of Ioannina, Ioannina, Greece

Theodora Tsikrika Center for Mathematics and Computer Science, Amsterdam, The Netherlands

Vassilis J. Tsotras University of California-Riverside, Riverside, CA, USA

Mikalai Tsytsarau University of Trento, Povo, Italy

Peter A. Tucker Whitworth University, Spokane, WA, USA

Anthony K. H. Tung National University of Singapore, Singapore, Singapore

Deepak Turaga IBM Research, San Francisco, CA, USA

Theodoros Tzouramanis University of the Aegean, Samos, Greece

Antti Ukkonen Helsinki University of Technology, Helsinki, Finland

Mollie Ullman-Cullere Harvard Medical School - Partners Healthcare Center for Genetics and Genomics, Boston, MA, USA

Ali Ünlï University of Augsburg, Augsburg, Germany

Antony Unwin Augsburg University, Augsburg, Germany

Susan D. Urban Arizona State University, Phoenix, AZ, USA

Jaideep Vaidya Rutgers University, Newark, NJ, USA

Alejandro A. Vaisman Instituto Tecnológico de Buenos Aires, Buenos Aires, Argentina

Shivakumar Vaithyanathan IBM Almaden Research Center, San Jose, CA, USA

Athena Vakali Aristotle University, Thessaloniki, Greece

Patrick Valduriez INRIA, LINA, Nantes, France

Maarten van Steen VU University, Amsterdam, The Netherlands

W. M. P. van der Aalst Eindhoven University of Technology, Eindhoven, The Netherlands

Christelle Vangenot EPFL, Lausanne, Switzerland

Stijn Vansummeren Hasselt University and Transnational University of Limburg, Diepenbeek, Belgium

Vasilis Vassalos Athens University of Economics and Business, Athens, Greece

Michael Vassilakopoulos University of Thessaly, Volos, Greece 
Panos Vassiliadis University of Ioannina, Ioannina, Greece

Michalis Vazirgiannis Athens University of Economics and Business, Athens, Greece

Olga Vechtomova University of Waterloo, Waterloo, ON, Canada

Erik Vee Yahoo! Research, Silicon Valley, CA, USA

Jari Veijalainen University of Jyvaskyla, Jyvaskyla, Finland

Yannis Velegrakis Department of Information Engineering and Computer Science, University of Trento, Trento, Italy

Suresh Venkatasubramanian University of Utah, Salt Lake City, UT, USA

Rossano Venturini Department of Computer Science, University of Pisa, Pisa, Italy

Victor Vianu University of California-San Diego, La Jolla, CA, USA

Maria-Esther Vidal Computer Science, Universidad Simon Bolivar, Caracas, Venezuela

Millist Vincent University of South Australia, Adelaide, SA, Australia

Giuliana Vitiello University of Salerno, Salerno, Italy

Michail Vlachos IBM T.J. Watson Research Center, Hawthorne, NY, USA

Akrivi Vlachou Athena Research and Innovation Center, Institute for the Management of Information Systems, Athens, Greece

Hoang Vo Computer Science, Stony Brook University, Stony Brook, NY, USA

Hoang Tam Vo IBM Research, Melbourne, VIC, Australia

Agnès Voisard Fraunhofer Institute for Software and Systems Engineering (ISST), Berlin, Germany

Kaladhar Voruganti Advanced Development Group, Network Appliance, Sunnyvale, CA, USA

Gottfried Vossen Department of Information Systems, Westfälische Wilhelms-Universität, Münster, Germany

Daisy Zhe Wang Computer and Information Science and Engineering (CISE), University of Florida, Gainesville, FL, USA

Feng Wang City University of Hong Kong, Hong Kong, China

Fusheng Wang Stony Brook University, Stony Brook, NY, USA

Jianyong Wang Tsinghua University, Beijing, China

Jun Wang Queen Mary University of London, London, UK

Meng Wang Microsoft Research Asia, Beijing, China 
X. Sean Wang School of Computer Science, Fudan University, Shanghai, China

Xin-Jing Wang Microsoft Research Asia, Beijing, China

Micros Facebook, CA, USA

Zhengkui Wang InfoComm Technology, Singapore Institute of Technology, Singapore, Singapore

Matthew O. Ward Worcester Polytechnic Institute, Worcester, MA, USA

Segev Wasserkrug IBM Research Labs-Haifa, Haifa, Israel

Hans Weda Phillips Research Europe, Eindhoven, The Netherlands

Gerhard Weikum Department 5: Databases and Information Systems, Max-Planck-Institut für Informatik, Saarbrücken, Germany

Michael Weiner Regenstrief Institute, Inc., Indiana University School of Medicine, Indianapolis, IN, USA

Michael Weiss Carleton University, Ottawa, ON, Canada

Ji-Rong Wen Microsoft Research Asia, Beijing, China

Chunhua Weng Columbia University, New York, NY, USA

Mathias Weske University of Potsdam, Potsdam, Germany

Thijs Westerveld Teezir Search Solutions, Ede, Netherlands

Till Westmann Oracle Labs, Redwood City, CA, USA

Karl Wiggisser Institute of Informatics-Systems, University of Klagenfurt, Klagenfurt, Austria

Jef Wijsen University of Mons, Mons, Belgium

Mark D. Wilkinson University of British Columbia, Vancouver, BC, Canada

Graham Wills SPSS Inc., Chicago, IL, USA

Ian H. Witten University of Waikato, Hamilton, New Zealand

Kent Wittenburg Mitsubishi Electric Research Laboratories, Inc., Cambridge, MA, USA

Eric Wohlstadter University of British Columbia, Vancouver, BC, Canada

Dietmar Wolfram University of Wisconsin-Milwaukee, Milwaukee, WI, USA

Ouri Wolfson Mobile Information Systems Center (MOBIS), The University of Illinois at Chicago, Chicago, IL, USA

Department of CS, University of Illinois at Chicago, Chicago, IL, USA

Janette Wong IBM Canada Ltd, Markham, ON, Canada 
Raymond Chi-Wing Wong Department of Computer Science and Engineering, The Hong Kong University of Science and Technology, Clear Water Bay, Kowloon, Hong Kong

Peter T. Wood Birkbeck, University of London, London, UK

David Woodruff IBM Almaden Research Center, San Jose, CA, USA

Marcel Worring University of Amsterdam, Amsterdam, The Netherlands

Adam Wright Partners HealthCare, Boston, MA, USA

Sai Wu Zhejiang University, Hangzhou, Zhejiang, People's Republic of China

Yuqing Wu Indiana University, Bloomington, IN, USA

Alex Wun University of Toronto, Toronto, ON, Canada

Ming Xiong Bell Labs, Murray Hill, NJ, USA

Google, Inc., New York, NY, USA

Guandong Xu University of Technology Sydney, Sydney, Australia

Hua Xu Columbia University, New York, NY, USA

Jun Yan Microsoft Research Asia, Haidian, China

Xifeng Yan IBM T. J. Watson Research Center, Hawthorne, NY, USA

Jun Yang Duke University, Durham, NC, USA

Li Yang Western Michigan University, Kalamazoo, MI, USA

Ming-Hsuan Yang University of California at Merced, Merced, CA, USA

Seungwon Yang Virginia Tech, Blacksburg, VA, USA

Yang Yang Center for Future Media and School of Computer Science and Engineering, University of Electronic Science and Technology of China, Chengdu, Sichuan, China

Yun Yang Swinburne University of Technology, Melbourne, VIC, Australia

Yu Yang City University of Hong Kong, Hong Kong, China

Yong Yao Cornell University, Ithaca, NY, USA

Mikalai Yatskevich University of Trento, Trento, Italy

Xun Yi Computer Science and Info Tech, RMIT University, Melbourne, VIC, Australia

Hiroshi Yoshida VLSI Design and Education Center, University of Tokyo, Tokyo, Japan

Fujitsu Limited, Yokohama, Japan

Masatoshi Yoshikawa University of Kyoto, Kyoto, Japan 
Matthew Young-Lai Sybase iAnywhere, Waterloo, ON, Canada

Google, Inc., Mountain View, CA, USA

Hwanjo Yu University of Iowa, Iowa City, IA, USA

Ting Yu North Carolina State University, Raleigh, NC, USA

Cong Yu Google Research, New York, NY, USA

Philip S. Yu Computer Science Department, University of Illinois at Chicago, Chicago, IL, USA

Jeffrey Xu Yu Department of Systems Engineering and Engineering Management, The Chinese University of Hong Kong, Hong Kong, China

Pingpeng Yuan Service Computing Technology and System Lab, Cluster and Grid Computing Lab, School of Computer Science and Technology, Huazhong University of Science and Technology, Wuhan, China

Vladimir Zadorozhny University of Pittsburgh, Pittsburgh, PA, USA

Matei Zaharia Douglas T. Ross Career Development Professor of Software Technology, MIT CSAIL, Cambridge, MA, USA

Ilya Zaihrayeu University of Trento, Trento, Italy

Mohammed J. Zaki Rensselaer Polytechnic Institute, Troy, NY, USA

Carlo Zaniolo University of California-Los Angeles, Los Angeles, CA, USA

Hugo Zaragoza Yahoo! Research, Barcelona, Spain

Stan Zdonik Brown University, Providence, RI, USA

Demetrios Zeinalipour-Yazti Department of Computer Science, Nicosia, Cyprus

Hans Zeller Hewlett-Packard Laboratories, Palo Alto, CA, USA

Pavel Zezula Masaryk University, Brno, Czech Republic

Cheng Xiang Zhai University of Illinois at Urbana-Champaign, Urbana, IL, USA

Aidong Zhang State University of New York, Buffalo, NY, USA

Benyu Zhang Microsoft Research Asia, Beijing, China

Donghui Zhang Paradigm4, Inc., Waltham, MA, USA

Dongxiang Zhang School of Computer Science and Engineering, University of Electronic Science and Technology of China, Sichuan, China

Ethan Zhang University of California, Santa Cruz, CA, USA 
Jin Zhang University of Wisconsin Milwaukee, Milwaukee, WI, USA

Kun Zhang Xavier University of Louisiana, New Orleans, LA, USA

Lei Zhang Microsoft Research Asia, Beijing, China

Lei Zhang Microsoft Research, Redmond, WA, USA

Li Zhang Peking University, Beijing, China

Meihui Zhang Information Systems Technology and Design, Singapore University of Technology and Design, Singapore, Singapore

Qing Zhang The Australian e-health Research Center, Brisbane, Australia

Rui Zhang University of Melbourne, Melbourne, VIC, Australia

Dataware Ventures, Tucson, AZ, USA

Dataware Ventures, Redondo Beach, CA, USA

Yanchun Zhang Victoria University, Melbourne, VIC, Australia

Yi Zhang Yahoo! Inc., Santa Clara, CA, USA

Yue Zhang University of Pittsburgh, Pittsburgh, PA, USA

Zhen Zhang University of Illinois at Urbana-Champaign, Urbana, IL, USA

Feng Zhao Microsoft Research, Redmond, WA, USA

Ying Zhao Tsinghua University, Beijing, China

Baihua Zheng Singapore Management University, Singapore, Singapore

Yi Zheng University of Ontario Institute of Technology, Oshawa, ON, Canada

Yu Zheng Data Management, Analytics and Services (DMAS) and Ubiquitous Computing Group (Ubicomp), Microsoft Research Asia, Beijing, China

Zhi-Hua Zhou National Key Lab for Novel Software Technology, Nanjing University, Nanjing, China

Jingren Zhou Alibaba Group, Hangzhou, China

Li Zhou Partners HealthCare System Inc., Boston, MA, USA

Xiaofang Zhou School of Information Technology and Electrical Engineering, University of Queensland, Brisbane, QLD, Australia

Huaiyu Zhu IBM Almaden Research Center, San Jose, CA, USA

Xiaofeng Zhu Guangxi Normal University, Guilin, Guangxi, People's Republic of China

Xingquan Zhu Florida Atlantic University, Boca Raton, FL, USA 
Cai-Nicolas Ziegler Siemens AG, Munich, Germany

Hartmut Ziegler University of Konstanz, Konstanz, Germany

Esteban Zimányi CoDE, Université Libre de Bruxelles, Brussels, Belgium

Arthur Zimek Ludwig-Maximilians-Universität München, Munich, Germany

Department of Mathematics and Computer Science, University of Southern Denmark, Odense, Denmark

Roger Zimmermann Department of Computer Science, School of Computing, National University of Singapore, Singapore, Republic of Singapore

Lei Zou Institute of Computer Science and Technology, Peking University, Beijing, China 